\title{
BRILL-NOETHER THEORY OF CURVES ON ENRIQUES SURFACES I: THE POSITIVE CONE AND GONALITY
}

\author{
ANDREAS LEOPOLD KNUTSEN* AND ANGELO FELICE LOPEZ** \\ $\dagger$ Dedicated to the memory of Silvano Bispuri
}

\begin{abstract}
We study the existence of linear series on curves lying on an Enriques surface and general in their complete linear system. Using a method that works also below the Bogomolov-Reider range, we compute, in all cases, the gonality of such curves. We also give a new result about the positive cone of line bundles on an Enriques surface and we show how this relates to the gonality.
\end{abstract}

\section{INTRODUCTION}

Let $S$ be a smooth surface and let $L$ be a line bundle on $S$. There is a natural interaction between the geometry of $S$ and that of the curves $C \in|L|$. On the one hand, strong geometric properties of curves in $|L|$, do lead, in many cases, to analogous properties of $S$ itself. This is the case for example if all smooth curves in $|L|$ are hyperelliptic ([Ca, En, [SV]), or trigonal $([\mathrm{Se}, \mathrm{R}, \mathrm{PaO}, \mathrm{Fa}])$, etc.. On the other hand one can choose an interesting type of surface and try to derive as much information as possible on the curves in $|L|$. Celebrated examples of this investigation range from well-known classical ones to very recent ones. Perhaps this line of thought was revived by the Green-Harris-Mumford conjecture, namely that all smooth curves in a given linear series on a K3 surface have the same Clifford index.

To study curves on K3 surfaces new interesting vector bundle methods were introduced by Lazarsfeld, Tyurin, Reider, Donagi and Morrison [DM], culminating on one side with the proof of the Green-Harris-Mumford conjecture GL and on the other with the fact that curves on a K3 surface of rank one do behave, from the point of view of Brill-Noether theory, like general ones [La].

The study of Brill-Noether theory of curves in a given complete linear system $|L|$ on a surface $S$ has, besides its own beauty, lots of interesting applications. We mention here the one that was our main motivation.

Suppose that $L$ is very ample, giving an embedding $S \subset \mathbb{P}^{r}=\mathbb{P} H^{0}(L)$. In the study of threefolds it is interesting to know whether there exists a threefold $X \subset \mathbb{P}^{r+1}$ different for a cone over $S$ and such that $S=X \cap \mathbb{P}^{r}$. If $r \geq 4$ there is a well-known condition (Zak's theorem [Za]): If $h^{0}\left(N_{S / \mathbb{P}^{r}}(-1)\right) \leq r+1$, where $N_{S / \mathbb{P}^{r}}$ is the normal bundle of $S$, then there is no such $X$. Now the cohomology of the normal bundle of $S$ is often related to the one of a smooth hyperplane section $Y=S \cap H$. But on a curve we have the formula ([Wa]) $h^{0}\left(N_{Y / \mathbb{P}^{r-1}}(-1)\right)=r+\operatorname{cork} \Phi_{H_{Y}, \omega_{Y}}$, where $\Phi_{H_{Y}, \omega_{Y}}$ is the Gaussian map associated to the canonical and hyperplane bundle $H_{Y}$ of $Y$. At last, the surjectivity of Gaussian maps on a curve $Y$ is very much governed by its Brill-Noether theory ([Wa, BEL]). Moreover, as the

\footnotetext{
* Research partially supported by a Marie Curie Intra-European Fellowship within the 6th European Community Framework Programme.

** Research partially supported by the MIUR national project "Geometria delle varietà algebriche" COFIN 2002-2004.

2000 Mathematics Subject Classification: Primary 14H51, 14C20, 14J28. Secondary 14J05, 14 F17.
} 
results of [KLM] show, this knowledge will be needed also when $Y$ is not necessarily the hyperplane section of $X$.

In the present article we investigate the Brill-Noether theory of curves on an Enriques surface, emphasizing the calculation of the gonality (see Thm. 1), prove a new result on the positive cone on an Enriques surface (see Prop. 1) and show how these two results are related. In a subsequent paper [KL2], we will study Clifford index and exceptional curves. These results will play a crucial role in [KLM], where, among other things, we prove the genus bound $g \leq 17$ for threefolds having an Enriques surface as hyperplane section.

Let now $S$ be an Enriques surface and let $|L|$ be a base-component free complete linear system on $S$. Unlike (most cases of) $K 3$ and Del Pezzo surfaces, it is not the case that the Clifford index or the gonality of smooth curves in $|L|$ are constant, as simple examples show. Therefore perhaps the best first question one can ask is about the linear series on general curves in $|L|$. Now recall from [CD]:

Definition 1.1. Let $L$ be a line bundle on an Enriques surface $S$ such that $L^{2}>0$. Set

$$
\phi(L):=\inf \left\{|F . L|: F \in \operatorname{Pic} S, F^{2}=0, F \not \equiv 0\right\} .
$$

An important property of this function, which will be used throughout the article, is that $\phi(L)^{2} \leq L^{2}$ [CD, Cor.2.7.1]. Hence, for $L^{2}>>0$, we have, for any smooth $C \in|L|$, that gon $C \leq 2 \phi(L)<<\left\lfloor\frac{L^{2}}{4}\right\rfloor+2=\left\lfloor\frac{g(C)+3}{2}\right\rfloor$, so that the curves are far from being Brill-Noether general, again unlike on (general) $K 3$ surfaces. One might expect that either the BrillNoether theory of general curves or elliptic pencils on the surface are enough to calculate the gonality of general curves in $|L|$, but this turns out to be false: Let $\left|2 E_{1}\right|,\left|2 E_{2}\right|$ be two genus one pencils such that $E_{1} \cdot E_{2}=2$ (they exist on a general Enriques surface by [Co1, Thm.2.7.2]) and let $L=n\left(E_{1}+E_{2}\right)$. We have $L^{2}=4 n^{2}$ and $\phi(L)=2 n$. Let $C \in|L|$ be a general curve and set $B=E_{1}+E_{2}$. For $n \geq 2$ we find that $\left|B_{\mid C}\right|$ is a $g_{4 n}^{2}$ that cannot be very ample, else $4 n^{2}=4 n(4 n-3)$, hence $n=1$. Therefore gon $(C) \leq 4 n-2<2 \phi(L)$.

Motivated by the above examples we give the ensuing

Definition 1.2. Let $L$ be a line bundle on an Enriques surface $S$ such that $L^{2}>0$. We define

$$
\mu(L)=\min \left\{B . L-2: B \in \operatorname{Pic}(S) \text { with } B \text { effective, } B^{2}=4, \phi(B)=2, B \not \equiv L\right\} .
$$

The first of the two main results of this paper shows that in fact the gonality of a general smooth curve in a given complete linear system on an Enriques surface is governed by elliptic pencils and divisors of self-intersection 4 :

\section{Theorem 1.}

Let $|L|$ be a base-component free complete linear system on an Enriques surface $S$ such that $L^{2}>0$. Then, for a general $C \in|L|$, we have

$$
\operatorname{gon}(C)=\min \left\{2 \phi(L), \mu(L),\left\lfloor\frac{L^{2}}{4}\right\rfloor+2\right\} .
$$

The question now arises of how to compute the "new" function $\mu(L)$. In section 2 we classify line bundles for which $\mu(L)<2 \phi(L)$ (Proposition 2.8). It turns out that such line bundles are the "extremal ones" in the positive cone in a way we now explain.

As mentioned above, $L^{2} \geq \phi(L)^{2}$ for any line bundle $L$ on an Enriques surface with $L^{2}>0$. We prove that there are no line bundles with $\phi(L)^{2}<L^{2}<\phi(L)^{2}+\phi(L)-2$ and we classify the borderline cases. (The proposition is stated for simplicity for $L$ effective, otherwise it will hold for $K_{S}-L$ by Riemann-Roch) 


\section{Proposition 1.}

Let $L$ be an effective line bundle on an Enriques surface with $L^{2}>0$. If $L^{2} \leq \phi(L)^{2}+$ $\phi(L)-2$, then there exist primitive effective divisors $E_{i}$ with $E_{i}^{2}=0$, for $i=1,2,3$, $E_{1} \cdot E_{2}=E_{1} \cdot E_{3}=2, E_{2} \cdot E_{3}=1$ and an integer $h \geq 1$ so that one of the two following occurs:

(i) $L^{2}=\phi(L)^{2}$. In this case $L \equiv h\left(E_{1}+E_{2}\right)$.

(ii) $L^{2}=\phi(L)^{2}+\phi(L)-2$. In this case either

(ii-a) $L \sim h\left(E_{1}+E_{2}\right)+E_{3}$; or

(ii-b) $L \sim(h+1) E_{1}+h E_{2}+E_{3}$; or

(ii-c) $L \equiv 2\left(E_{1}+E_{2}+E_{3}\right)$ (whence $L^{2}=40$ and $\phi(L)=6$ ).

The relation to the function $\mu(L)$ is that the line bundles appearing in (i), (ii-a) and (ii-b) are precisely the ones for which $\mu(L)<2 \phi(L)$ (Proposition 2.8). In other words, linear systems whose general members have "nongeneral" (in the sense of Brill-Noether theory) gonalities not computed by elliptic pencils are precisely the "extremal" cases (i), (ii-a) and (ii-b) in the positive cone.

In light of this, Theorem 1 can be stated only in terms of $L^{2}$ and $\phi(L)$ :

\section{Corollary 1.}

Let $|L|$ be a base-component free complete linear system on an Enriques surface such that $L^{2}>0$ and let $C \in|L|$ be a general curve. Then

$$
\operatorname{gon}(C)=2 \phi(L)
$$

unless $L$ is of one of the following types:

(a) $L^{2}=\phi(L)^{2}$ with $\phi(L) \geq 2$ and even. In these cases $\operatorname{gon}(C)=2 \phi(L)-2$.

(b) $L^{2}=\phi(L)^{2}+\phi(L)-2$ with $\phi(L) \geq 3, L \not \equiv 2 D$ for $D$ such that $D^{2}=10, \phi(D)=3$. In these cases $\operatorname{gon}(C)=2 \phi(L)-1$ except for $\phi(L)=3,4$ when $\operatorname{gon}(C)=2 \phi(L)-2$.

(c) $\left(L^{2}, \phi(L)\right)=(30,5),(22,4),(20,4),(14,3),(12,3)$ and $(6,2)$. In these cases $\operatorname{gon}(C)=\left\lfloor\frac{L^{2}}{4}\right\rfloor+2=2 \phi(L)-1$.

The line bundles in (a), (b) and (c) above have an explicit description by Proposition 1.

We also obtain the following result about the variation of the gonality of smooth curves in a complete linear system:

\section{Corollary 2.}

Let $|L|$ be a base-component free complete linear system on an Enriques surface such that $L^{2}>0$. Let gengon $|L|$ denote the gonality of a general smooth curve in $|L|$ and mingon $|L|$ denote the minimal gonality of a smooth curve in $|L|$. Then

$$
\text { gengon }|L|-2 \leq \text { mingon }|L| \leq \text { gengon }|L| \text {. }
$$

Moreover if equality holds on the left, then $\phi(L) \geq\left\lceil\sqrt{\frac{L^{2}}{2}}\right\rceil$.

Finally in 6.1 we give examples showing that this result is sharp, that is that all the cases mingon $|L|=$ gengon $|L|-2$, gengon $|L|-1$ and gengon $|L|$ do occur.

Aside from our use of well-known vector bundle methods, to study linear series on curves on an Enriques surface we will proceed, in section 4, as follows. If a general curve $C \in|L|$ carries some "unexpected" linear series, then it also carries some zero-dimensional schemes not imposing independent conditions on some subbundle of $L$. By moving these schemes on $S$ we will often derive a contradiction or find that the gonality is computed by $\mu(L)$. An important feature of this method is that, unlike all the previous ones, it does work well, 
in many cases, also below the Bogomolov-Reider range, that is when $L^{2}<4 \operatorname{gon}(C)$ (see Proposition 4.6), and also for other types of surfaces besides Enriques surfaces.

Acknowledgments. We wish to thank Roberto Muñoz for several helpful discussions.

\section{Basic Results on line bundles on EnRiques surfaces}

Definition 2.1. We denote by $\sim$ (resp. $\equiv$ ) the linear (resp. numerical) equivalence of divisors or line bundles. A line bundle $L$ is primitive if $L \equiv k L^{\prime}$ implies $k= \pm 1$. We will write $L \geq 0$ (respectively $L>0$ ) for an effective (resp. effective non trivial) line bundle $L$. If $V \subseteq H^{0}(L)$ is a linear system, we denote its base scheme by $\mathrm{Bs}|V|$. We denote by $|L|_{\text {sm }}$ the open subset of smooth curves in $|L|$. A nodal curve on an Enriques surface $S$ is a smooth rational curve contained in $S$.

We will often use another property of the function $\phi(L)$ defined in the introduction: If $L$ is nef, then there exists a nef divisor $E$ calculating $\phi(L)$ ([Co2, 2.11] or by [CD, Cor.2.7.1, Prop.2.7.1 and Thm.3.2.1]).

We also recall the following simple consequence of the signature theorem [BPV, VIII.1].

Lemma 2.2. KL1, Lemma 2.1] Let $X$ be a smooth surface and let $A>0$ and $B>0$ be divisors on $X$ such that $A^{2} \geq 0$ and $B^{2} \geq 0$. Then $A . B \geq 0$ with equality if and only if there exists a primitive divisor $F>0$ and integers $a \geq 1, b \geq 1$ such that $F^{2}=0$ and $A \equiv a F, B \equiv b F$.

This gives

Lemma 2.3. Let $L>0$ and $\Delta>0$ be divisors on an Enriques surface $S$ with $L^{2} \geq 0$, $\Delta^{2}=-2$ and $k:=-\Delta . L>0$. Then there exists an $A>0$ such that $A^{2}=L^{2}, A . \Delta=k$ and $L \sim A+k \Delta$. Moreover, if $L$ is primitive, then so is $A$.

Proof. Set $A=L-k \Delta$. Then $A . \Delta=k$ and $A^{2}=L^{2} \geq 0$. If $K_{S}-A \geq 0$, Lemma 2.2 yields $0 \leq\left(K_{S}-A\right) \cdot L=-L^{2}-k^{2}$, a contradiction. Hence $H^{2}(A)=0$ and by Riemann-Roch we get $A>0$. Now if $A \equiv q B$ with $q \geq 2$, then $k=q B . \Delta$, whence $L \equiv q(B+(B . \Delta) \Delta)$ is not primitive.

Lemma 2.4. Let $S$ be an Enriques surface and let $L$ be a line bundle on $S$ such that $L>0, L^{2}>0$. Let $F>0$ be a divisor on $S$ such that $F^{2}=0$ and $\phi(L)=|F . L|$. Then $F . L>0$ and if $\alpha>0$ is such that $(L-\alpha F)^{2} \geq 0$, then $L-\alpha F>0$.

Proof. By Lemma 2.2 we get F.L $>0$. If $(L-\alpha F)^{2} \geq 0$ we get by Riemann-Roch that either $L-\alpha F>0$ or $K_{S}-L+\alpha F>0$. But in the latter case, Lemma 2.2 gives the contradiction $-\phi(L)=F \cdot\left(K_{S}-L+\alpha F\right) \geq 0$.

We recall here a consequence of the vanishing theorem proved in KL1] that will be used throughout the article.

Definition 2.5. An effective line bundle $L$ on a $K 3$ or Enriques surface is said to be quasi-nef if $L^{2} \geq 0$ and $L . \Delta \geq-1$ for every $\Delta$ such that $\Delta>0$ and $\Delta^{2}=-2$.

Theorem 2.6. [KL1, Corollary 2.5] An effective line bundle $L$ on a $K 3$ or Enriques surface is quasi-nef if and only if $L^{2} \geq 0$ and either $h^{1}(L)=0$ or $L \equiv n E$ for some $n \geq 2$ and some primitive and nef divisor $E>0$ with $E^{2}=0$. 
2.1. Line bundles with $\mu(L)<2 \phi(L)$. We want to prove a result about the function $\mu(L)$. In fact, the cases for which $\mu(L)<2 \phi(L)$ are of a very particular type and we will classify them.

Definition 2.7. Let $E_{1}, E_{2}, E_{3}$ be three primitive divisors on an Enriques surface $S$ such that $E_{i}>0, E_{i}^{2}=0, i=1,2,3, E_{1} \cdot E_{2}=E_{1} \cdot E_{3}=2$ and $E_{2} \cdot E_{3}=1$.

A line bundle $L$ on $S$ is said to be of type

$\left(\mu_{1}\right)$ if $L \equiv h\left(E_{1}+E_{2}\right), h \geq 1$,

$\left(\mu_{2}\right)$ if $L \sim h\left(E_{1}+E_{2}\right)+E_{3}, h \geq 1$,

$\left(\mu_{3}\right)$ if $L \sim(h+1) E_{1}+h E_{2}+E_{3}, h \geq 1$.

The properties of these three line bundle types will be proved in Lemma 2.14 .

Our goal here is to prove the ensuing

Proposition 2.8. Let $L>0$ be a line bundle on an Enriques surface $S$ such that $L^{2}>0$ and $\left(L^{2}, \phi(L)\right) \neq(4,2)$. Then $\mu(L)<2 \phi(L)$ if and only if $L$ is of type $\left(\mu_{1}\right)$, $\left(\mu_{2}\right)$ or $\left(\mu_{3}\right)$.

Remark 2.9. The above proposition also holds when $\left(L^{2}, \phi(L)\right)=(4,2)$. In fact in this case we can prove that $\mu(L)=3$. However the proof of this is quite long and will be omitted, as we do not need it for the sequel.

To prove the proposition we first need a few auxiliary results.

Lemma 2.10. Let $D>0$ be a divisor on an Enriques surface $S$ such that $D^{2}=10$ and $\phi(D)=3$. Then there are ten divisors $F_{i}$ such that $F_{i}>0, F_{i}^{2}=0$ for $1 \leq i \leq 10$, $F_{i} . F_{j}=1$ for $1 \leq i<j \leq 10$ and $3 D \sim F_{1}+\ldots+F_{10}$. Moreover if $F$ and $F^{\prime}$ satisfy $F>0$, $F^{\prime}>0, F^{2}=\left(F^{\prime}\right)^{2}=0, F \cdot D=F^{\prime} . D=3$ and $F \not \equiv F^{\prime}$, then $F \cdot F^{\prime}=1$.

Proof. The first assertion easily follows from [CD, Cor.2.5.5], together with Lemma 2.2 for the effectiveness of the $F_{i}$. For the last assertion, $F .(3 D)=9$ implies that $F \cdot F_{j}=0$ for some $j$, whence $F \equiv F_{j_{1}}, F^{\prime} \equiv F_{j_{2}}$ with $j_{1} \neq j_{2}$ by Lemma 2.2, so that $F . F^{\prime}=1$.

Lemma 2.11. Let $L>0$ be a line bundle on an Enriques surface $S$ such that $L^{2}>0$. Then $L^{2}=\phi(L)^{2}$ if and only if $L$ is of type $\mu_{1}$.

Proof. The "if" part is immediate. For the converse, assume $L^{2}=\phi(L)^{2}$. Since $L^{2}$ is even, $\phi(L)$ must be even, say $\phi(L)=2 h$, so that $L^{2}=4 h^{2}$. The result is clear for $h=1$, so we assume henceforth that $h \geq 2$. Pick any $E_{1}>0$ with $E_{1}^{2}=0$ and $E_{1} \cdot L=2 h$. Set $L_{1}=L-E_{1}$, so that $L_{1}^{2}=4 h(h-1)$ and $L_{1}>0$ by Lemma 2.4 .

If $\phi\left(L_{1}\right) \geq 2 h-1$, then $4 h^{2}-4 h=L_{1}^{2} \geq \phi\left(L_{1}\right)^{2} \geq(2 h-1)^{2}$, a contradiction. Therefore $\phi\left(L_{1}\right) \leq 2 h-2$. Pick any $E_{2}>0$ with $E_{2}^{2}=0$ and $E_{2} \cdot L_{1}=\phi\left(L_{1}\right)$. Then $2 h-E_{1} \cdot E_{2} \leq$ $E_{2} \cdot L-E_{2} \cdot E_{1}=E_{2} \cdot L_{1} \leq 2 h-2$, whence $E_{1} \cdot E_{2} \geq 2$ and if equality holds we have that $E_{2} . L=2 h$. Now $\left(E_{1}+E_{2}\right) \cdot L \leq 4 h-2+E_{1} \cdot E_{2}$, whence $\left(L_{1}-E_{2}\right)^{2} \geq 4(h-1)^{2} \geq 4$ and $L_{1}-E_{2}>0$ by Lemma 2.4. The Hodge index theorem on $E_{1}+E_{2}$ and $L$ yields that either $E_{1} \cdot E_{2}=2$ or $E_{1} \cdot E_{2}-2 \geq 8 h(h-1)$. In the latter case we have $E_{1} \cdot\left(L_{1}-E_{2}\right) \leq$ $2 h-8 h(h-1)-2<0$, contradicting Lemma 2.2 .

Hence $E_{1} \cdot E_{2}=2, E_{1} \cdot L=E_{2} \cdot L=2 h$ and we are done by the Hodge index theorem.

Now we need to prove an integer version of [Co2, Lemma 1.4.2].

Lemma 2.12. Let $L>0$ be a line bundle on an Enriques surface $S$ with $L^{2} \geq 0$. Then there is an integer $n$ such that $1 \leq n \leq 10$ and, for every $i=1, \ldots, n$, there are primitive divisors $E_{i}>0$ with $E_{i}^{2}=0$ and integers $a_{i}>0$ such that

$$
L \equiv a_{1} E_{1}+\ldots+a_{n} E_{n}
$$

and one of the three following intersection sets occurs: 
(i) $E_{i} \cdot E_{j}=1$ for $1 \leq i<j \leq n$.

(ii) $n \geq 2, E_{1} \cdot E_{2}=2$ and $E_{i} \cdot E_{j}=1$ for $2 \leq i<j \leq n$ and for $i=1,3 \leq j \leq n$.

(iii) $n \geq 3, E_{1} \cdot E_{2}=E_{1} \cdot E_{3}=2$ and $E_{i} \cdot E_{j}=1$ for $3 \leq i<j \leq n$, for $i=1,4 \leq j \leq n$ and for $i=2,3 \leq j \leq n$.

Proof. We prove the lemma by induction on $L^{2}$. Since the case $L^{2}=0$ is obvious, we assume $L^{2} \geq 2$. By Lemma 2.4 we can choose a primitive divisor $F>0$ with $F^{2}=0, F . L=\phi(L)$ and, setting $L_{1}=L-F$, we get that $0 \leq L_{1}^{2}<L^{2}$ and $L_{1}>0$. By induction, we have that $L_{1} \equiv a_{1} E_{1}+\ldots+a_{n} E_{n}$ and $L \equiv F+a_{1} E_{1}+\ldots+a_{n} E_{n}$ with the intersections among the $E_{i}$ 's as in (i), (ii) or (iii). Note that if $F . E_{i} \leq 1$ for all $i=1, \ldots, n$ then, by Lemma 2.2, we have that either $F \equiv E_{i}$ for some $i$ and then $L$ has the desired decomposition or $F . E_{i}=1$ for all $i$. In the latter case we cannot have $n=10$ because the intersection matrix of $F, E_{1}, \ldots, E_{10}$ has nonzero determinant while the Enriques lattice has rank 10. Hence $n \leq 9$ and we are done if $F . E_{i} \leq 1$ for all $i=1, \ldots, n$.

Therefore we will henceforth assume that $F . E_{i} \geq 1$ for all $i=1, \ldots, n$ and that there is an index $i_{0}$ such that F. $E_{i_{0}} \geq 2$.

We divide the proof in the three cases corresponding to the intersections of the $E_{i}$ 's. To simplify some computations we set $a:=\sum_{i=1}^{n} a_{i}$.

Case 1: $E_{i} \cdot E_{j}=1$ for $1 \leq i<j \leq n$.

If $n \geq 2$ pick $j \in\{1, \ldots, n\}-\left\{i_{0}\right\}$. Then $E_{j} . L=E_{j} . F+a-a_{j} \geq \phi(L)=F . L \geq$ $a-a_{j}-a_{i_{0}}+a_{i_{0}} E_{i_{0}} . F+a_{j} E_{j} . F \geq a-a_{j}+a_{i_{0}}+a_{j} E_{j} . F$ giving the contradiction $0 \geq$ $a_{i_{0}}+\left(a_{j}-1\right) E_{j} . F \geq 1$. Therefore $n=i_{0}=1$ and $E_{1} \cdot F=E_{1} \cdot L \geq \phi(L)=F \cdot L=a_{1} E_{1} \cdot F$ that is $a_{1}=1$. Now $\phi(L)^{2}=\left(E_{1} \cdot F\right)^{2} \leq L^{2}=2 E_{1} \cdot F$, whence $E_{1} \cdot F=2$ and we are done for Case 1.

Case 2: $n \geq 2, E_{1} \cdot E_{2}=2$ and $E_{i} \cdot E_{j}=1$ for $2 \leq i<j \leq n$ and for $i=1,3 \leq j \leq n$.

If $n \geq 3$ we have $E_{3} \cdot L=E_{3} \cdot F+a-a_{3} \geq \phi(L)=F . L=\sum_{i=1}^{n} a_{i} E_{i} . F$. If there is an $i_{1} \in\{1, \ldots, n\}-\{3\}$ such that F. $E_{i_{1}} \geq 2$ then, as above, F.L $\geq a-a_{3}+a_{i_{1}}+a_{3} E_{3} . F$, giving the contradiction $0 \geq a_{i_{1}}+\left(a_{3}-1\right) E_{3} . F \geq 1$. Therefore we have that $F . E_{i}=1$ for all $i \in\{1, \ldots, n\}-\{3\}$ and $i_{0}=3$. Then $E_{3} . L=E_{3} . F+a-a_{3} \geq F \cdot L \geq a_{3} E_{3} . F+a-a_{3}$, whence $a_{3}=1$. Now $E_{1} \cdot L=1+a-a_{1}+a_{2} \geq F \cdot L \geq a+1$ and $E_{2} \cdot L=1+a-a_{2}+a_{1} \geq F . L \geq a+1$, giving $a_{1}=a_{2}$ and $E_{3} \cdot F=2$. If $n \geq 4$ we have $E_{4} \cdot L=1+a-a_{4} \geq F$.L $=a+1$, a contradiction. Hence $n=3$ and $\phi(L)=2 a_{1}+2, L^{2}=4\left(a_{1}+1\right)^{2}$. Therefore $L^{2}=\phi(L)^{2}$ and we are done by Lemma 2.11 .

If $n=2$ we suppose, without loss of generality, that $E_{2} . F \geq 2$. From $E_{i} . L \geq F . L$, $i=1,2$, we get $0 \geq\left(a_{1}-1\right) E_{1} \cdot F+a_{2}\left(E_{2} \cdot F-2\right)$ and $0 \geq\left(a_{2}-1\right) E_{2} \cdot F+a_{1}\left(E_{1} \cdot F-2\right)$. The first inequality gives $a_{1}=1, E_{2} \cdot F=2$ and the second becomes $0 \geq 2\left(a_{2}-1\right)+\left(E_{1} \cdot F-2\right)$. If $E_{1} \cdot F \geq 2$ we get that also $a_{2}=1, E_{1} \cdot F=2$, whence $\phi(L)=F \cdot L=4$ while $L^{2}=12$, a contradiction. Therefore $E_{1} \cdot F=1$ and we are done in this case.

Case 3: $n \geq 3, E_{1} \cdot E_{2}=E_{1} \cdot E_{3}=2$ and $E_{i} \cdot E_{j}=1$ for $3 \leq i<j \leq n$, for $i=1$, $4 \leq j \leq n$ and for $i=2,3 \leq j \leq n$.

If $n \geq 4$ we have $E_{4} \cdot L=E_{4} \cdot F+a-a_{4} \geq \phi(L)=F . L=\sum_{i=1}^{n} a_{i} E_{i} . F$. If there is an $i_{1} \in\{1, \ldots, n\}-\{4\}$ such that $F . E_{i_{1}} \geq 2$ then, as above, F.L $\geq a-a_{4}+a_{i_{1}}+a_{4} E_{4} . F$, giving the contradiction $0 \geq a_{i_{1}}+\left(a_{4}-1\right) E_{4} \cdot F \geq 1$. Therefore we have that $F \cdot E_{i}=1$ for all $i \in\{1, \ldots, n\}-\{4\}$ and $i_{0}=4$. Then $E_{4} \cdot\left(E_{1}+E_{2}+E_{3}\right)=F \cdot\left(E_{1}+E_{2}+E_{3}\right)=3$, whence $E_{4} . F=1$ by Lemma 2.10, a contradiction. 
Hence $n=3$ and from $E_{i} . L \geq F . L, i=1,2,3$, we get

$$
\begin{aligned}
& 0 \geq\left(a_{1}-1\right) E_{1} \cdot F+a_{2}\left(E_{2} \cdot F-2\right)+a_{3}\left(E_{3} \cdot F-2\right), \\
& 0 \geq a_{1}\left(E_{1} \cdot F-2\right)+\left(a_{2}-1\right) E_{2} \cdot F+a_{3}\left(E_{3} \cdot F-1\right), \\
& 0 \geq a_{1}\left(E_{1} \cdot F-2\right)+a_{2}\left(E_{2} \cdot F-1\right)+\left(a_{3}-1\right) E_{3} \cdot F .
\end{aligned}
$$

Now if $E_{1} \cdot F \geq 2$ we get from (3) that $E_{1} \cdot F=2, E_{2} \cdot F=a_{3}=1$. Then (2) gives $E_{3} \cdot F=$ $a_{2}=1$ and (11) implies that $1 \leq a_{1} \leq 2$. Then $\phi(L)=F . L=2 a_{1}+2$ while $L^{2}=12 a_{1}+6$. This gives a contradiction when $a_{1}=2$ since then $\phi(L)=6$ and $L^{2}=30<36$. Therefore $a_{1}=1, \phi(L)=4$ and $L^{2}=18$. Now $(L-2 F)^{2}=2$ whence, by Lemma 2.4, we can write $L-2 F \sim F_{1}+F_{2}$ with $F_{i}>0, F_{i}^{2}=0, i=1,2$ and $F_{1} \cdot F_{2}=1$. Also $4 \leq F_{i} \cdot L=2 F_{i} \cdot F+1$, therefore $F_{i} . F \geq 2$ for $i=1,2$. Since $F_{1} \cdot F+F_{2} \cdot F=F .(L-2 F)=4$ we get $F_{1} \cdot F=F_{2} \cdot F=2$ and we are done in this case.

Therefore we can assume in the sequel of the proof that $E_{1} \cdot F=1$.

Now if $E_{i} \cdot F \geq 2$ for $i=2,3$ we get from (1) that $E_{2} \cdot F=E_{3} \cdot F=2$ and $a_{1}=1$. Adding up (2) and (3) gives $0 \geq-6+3 a_{2}+3 a_{3} \geq 0$, therefore also $a_{2}=a_{3}=1$. But then $\phi(L)=F \cdot L=5$ and $L^{2}=20$, a contradiction.

Therefore we can assume, without loss of generality, that $E_{1} \cdot F=E_{2} \cdot F=1, E_{3} \cdot F \geq 2$.

Now (1) becomes $a_{2} \geq a_{1}+a_{3}\left(E_{3} \cdot F-2\right)-1$ and (2) becomes $a_{1} \geq a_{2}+a_{3}\left(E_{3} \cdot F-1\right)-1$. Combining we get $a_{3}\left(2 E_{3} \cdot F-3\right) \leq 2$, whence $E_{3} \cdot F=2$ and $1 \leq a_{3} \leq 2$. Using again the inequalities (11) and (2) we get the only possibilities $a_{3}=2, a_{2}=a_{1}-1$ or $a_{3}=1, a_{1}-1 \leq$ $a_{2} \leq a_{1}$. In the first case we get $\phi(L)^{2}=(F . L)^{2}=\left(2 a_{1}+3\right)^{2}>L^{2}=4 a_{1}^{2}+12 a_{1}+2$, a contradiction. In the second case, setting $b=a_{1}$, we have the two possibilities

$$
\begin{gathered}
L \equiv F+b E_{1}+b E_{2}+E_{3} \\
L \equiv F+b E_{1}+(b-1) E_{2}+E_{3} .
\end{gathered}
$$

Set $D=E_{1}+E_{2}+E_{3}$ so that $D^{2}=10$ and $\phi(D)=3$. We can write $3 D \sim F_{1}+\ldots+F_{10}$ as in Lemma 2.10, Since $E_{2} \cdot D=E_{3} \cdot D=3$ by Lemma 2.2 we can assume, without loss of generality, that $E_{2} \equiv F_{1}, E_{3} \equiv F_{2}$, whence $E_{2} \cdot F_{i}=E_{3} \cdot F_{i}=1$ for $3 \leq i \leq 10$. Also $F . D=4$, therefore, by Lemma 2.2, $F . F_{i} \geq 1$ for $1 \leq i \leq 10$, and we can also assume, without loss of generality, that $F \cdot F_{3}=2$. Also from $12=3 E_{1} \cdot D=4+E_{1} \cdot F_{3}+\ldots+E_{1} \cdot F_{10}$ we see that $E_{1} \cdot F_{3}=1$. Now set $D^{\prime}=F+F_{3}+E_{3}$ so that $\left(D^{\prime}\right)^{2}=10$ and D.D $D^{\prime}=10$, therefore $D \equiv D^{\prime}$ by the Hodge index theorem. It follows that $E_{1}+E_{2} \equiv F+F_{3}$.

If $L$ is as in (4) then $L \equiv(b+1) F+b F_{3}+E_{3}$ with $F \cdot F_{3}=F \cdot E_{3}=2, F_{3} \cdot E_{3}=1$, has the required decomposition.

Now suppose that $L$ is as in (5). Set $F^{\prime}=L-b F-b F_{3}$. Now $\left(F^{\prime}\right)^{2}=0, F \cdot F^{\prime}=1$ whence $F^{\prime}>0$ by Riemann-Roch. Also $F_{3} \cdot F^{\prime}=2$ therefore $L \sim b F+b F_{3}+F^{\prime}$ with $F_{3} \cdot F=F_{3} \cdot F^{\prime}=2, F \cdot F^{\prime}=1$, has the required decomposition.

Lemma 2.13. Let $L>0$ be a line bundle on an Enriques surface $S$ with $L^{2}>0$. Then $\mu(L) \geq 2 \phi(L)-2$. Moreover if $\mu(L)<2 \phi(L)$ and $B$ is a line bundle computing $\mu(L)$, that is $B>0, B^{2}=4, \phi(B)=2, B \neq L$ and $B . L=\mu(L)$, then $B \sim F_{1}+F_{2}$, with $F_{i}>0$ primitive, $F_{i}^{2}=0, i=1,2, F_{1} \cdot F_{2}=2, F_{1} \cdot L=\phi(L)$ and $F_{2} \cdot L=\phi(L)$ or $\phi(L)+1$.

Proof. Use Lemma 2.4.

\section{Lemma 2.14.}

(i) If $L$ is of type $\left(\mu_{1}\right)$ then $\phi(L)=2 h$ and $L^{2}=\phi(L)^{2}$. If $h \geq 2$ then $\mu(L)=2 \phi(L)-2$.

(ii) If $L$ is of type $\left(\mu_{2}\right)$ then $\phi(L)=2 h+1, \mu(L)=2 \phi(L)-1$ and $L^{2}=\phi(L)^{2}+\phi(L)-2$.

(iii) If $L$ is of type $\left(\mu_{3}\right)$ then $\phi(L)=2 h+2, \mu(L)=2 \phi(L)-1$ and $L^{2}=\phi(L)^{2}+\phi(L)-2$. 
Proof. Apply Lemmas 2.10, 2.11 and 2.13.

Proof of Proposition 2.8. By Lemma 2.14 we can assume that $L^{2}>0,\left(L^{2}, \phi(L)\right) \neq(4,2)$ and $\mu(L)<2 \phi(L)$. We want to show that $L$ must be of type $\left(\mu_{1}\right),\left(\mu_{2}\right)$ or $\left(\mu_{3}\right)$. To do this, we divide the treatment into the three cases occurring in Lemma 2.12.

We set $a=\sum_{i=1}^{n} a_{i}$ and we choose $B \sim F_{1}+F_{2}$ as in Lemma 2.13 that computes $\mu(L)$, so that $F_{1} \cdot L=\phi(L)$ and $F_{2} \cdot L=\phi(L)$ or $\phi(L)+1$.

If $L$ is as in (i) of Lemma 2.12, then reordering the $a_{i}$ 's so that $a_{1} \geq \ldots \geq a_{n}$ we have $a-a_{1}=E_{1} . L \leq \ldots \leq E_{n} . L=a-a_{n}$. Now any $F>0$ with $F^{2}=0, F \not \equiv E_{i}$ for all i, satisfies $F . L \geq a$. Hence $\phi(L)=a-a_{1}<a$. Now $F_{1} . L=a-a_{1}$, whence we can assume $F_{1} \equiv E_{1}$ after renumbering indices. Since $F_{1} \cdot F_{2}=2$ we have $F_{2} \not \equiv E_{i}$ for $i \geq 2$, whence $F_{2} . L \geq a+a_{1}$, so that $\mu(L)=B . L-2 \geq 2(a-1) \geq 2 \phi(L)$, a contradiction.

If $L$ is as in (ii) of Lemma 2.12 we are done if $n=2$ and $a_{1}=a_{2}$. We assume that this is not the case. Reordering the $a_{i}$ 's so that $a_{1} \geq a_{2}$ and $a_{3} \geq \ldots \geq a_{n}$ we have $a+a_{2}-a_{1}=E_{1} \cdot L \leq E_{2} \cdot L=a+a_{1}-a_{2}$ and $a-a_{3}=E_{3} . L \leq \ldots \leq E_{n} . L=a-a_{n}$. Now any $F>0$ with $F^{2}=0, F \not \equiv E_{i}$ for all $i$, satisfies $F . L \geq a$. Hence $\phi(L)=E_{1} . L=a+a_{2}-a_{1} \leq a$ if $n=2$ and $\phi(L)=\min \left\{E_{1} \cdot L, E_{3} \cdot L\right\}=\min \left\{a+a_{2}-a_{1}, a-a_{3}\right\}<a$ if $n \geq 3$. Since $F_{1} . L=\phi(L)$, we can assume, after renumbering indices, that $F_{1} \equiv E_{1}$ or $F_{1} \equiv E_{3}$.

If $F_{1} \equiv E_{1}$, then $\phi(L)=a+a_{2}-a_{1}$ and either $F_{2} \equiv E_{2}$ or $F_{2} \not \equiv E_{i}$ for all $i$. In the first case, by Lemma 2.13, we have $2\left(a+a_{2}-a_{1}\right)+1=2 \phi(L)+1 \geq\left(F_{1}+F_{2}\right) \cdot L=\left(E_{1}+E_{2}\right) \cdot L=$ $2 a$, whence $a_{1}=a_{2}$ and $n=2$, a contradiction. In the second case we have $F_{2} . L \geq a+a_{1}$, whence $2\left(a+a_{2}-a_{1}\right)+1=2 \phi(L)+1 \geq\left(F_{1}+F_{2}\right) \cdot L=\left(E_{1}+F_{2}\right) \cdot L \geq 2 a+a_{2}$, therefore $a_{2}+1 \geq 2 a_{1} \geq 2 a_{2}$, that gives $a_{1}=a_{2}=1$. Then $\phi(L)=a$, whence $n=2$, again excluded.

If $F_{1} \equiv E_{3}$, then $\phi(L)=a-a_{3}<a$ and $F_{2} \not \equiv E_{i}$ for all $i$. Therefore $F_{2} . L \geq a+a_{3} \geq$ $\phi(L)+2$, contradicting Lemma 2.13 ,

Finally, if $L$ is as in (iii) of Lemma 2.12, we claim that we can write

$$
\begin{gathered}
L \equiv a_{1} E_{1}+\ldots+a_{10} E_{10}, \text { with } a_{1}>0, a_{2} \geq a_{3}>0, a_{4} \geq \ldots \geq a_{10} \geq 0 \\
E_{1} \cdot E_{2}=E_{1} \cdot E_{3}=2 \text { and } E_{i} \cdot E_{j}=1 \text { for }(i, j) \neq(1,2),(2,1),(1,3),(3,1) .
\end{gathered}
$$

Indeed, if $n=10$, this is clear after renumbering indices. If $3 \leq n<10$, we note that $D:=E_{1}+E_{2}+E_{3}$ satisfies $D^{2}=10$ and $\phi(D)=3$, so that $3 D \sim F_{1}+\ldots+F_{10}$ as in Lemma 2.10. Since $E_{i} \cdot(3 D)=9$ for all $i=2, \ldots, n$ by assumption, we must have $E_{i} \cdot F_{j}=0$ for some $j \in\{1, \ldots, 10\}$ for any $i=2, \ldots, n$, whence for $2 \leq i \leq n$ we have $E_{i}$ or $E_{i}+K_{S} \in\left\{F_{1}, \ldots, F_{10}\right\}$, so that each $F_{i} \not \equiv E_{j}$ for $j=2,3$, satisfies $F_{i} . E_{1}=1$. Therefore we can complete the set $\left\{E_{1}, \ldots, E_{n}\right\}$ to a set $\left\{E_{1}, \ldots, E_{n}, E_{n+1}, \ldots, E_{10}\right\}$ satisfying the desired conditions (setting $a_{n+1}=\ldots=a_{10}=0$ ).

Now, using (6), we deduce $a-a_{4}=E_{4} \cdot L \leq \ldots \leq E_{10} \cdot L=a-a_{10}, a-a_{2}+a_{1}=E_{2} \cdot L \leq$ $a-a_{3}+a_{1}=E_{3} . L$ and $E_{1} \cdot L=a+a_{2}+a_{3}-a_{1}$. Moreover, for any $F>0$ with $F^{2}=0$ and $F \not \equiv E_{i}, 1 \leq i \leq 10$, we have $F . L \geq a$. Combining the above we see that

$$
\phi(L)=\min \left\{E_{1} \cdot L, E_{2} \cdot L, E_{4} \cdot L\right\}=\min \left\{a+a_{2}+a_{3}-a_{1}, a-a_{2}+a_{1}, a-a_{4}\right\} .
$$

Next we will prove that $\mu(L) \geq 2 a+a_{3}-2$. Suppose then that $\mu(L)<2 a+a_{3}-2$.

Assume first that $F_{i} \not \equiv E_{j}$ for $i=1,2$ and all $j$. Then $F_{i} . L \geq a$ and using (77) we have

$$
2 a-2 \leq \mu(L)<2 \phi(L)=\min \left\{2\left(a+a_{2}+a_{3}-a_{1}\right), 2\left(a-a_{2}+a_{1}\right), 2\left(a-a_{4}\right)\right\} .
$$

It follows that $a_{4}=0$ (whence $a_{5}=\ldots=a_{10}=0$ ) and

$$
a_{2} \leq a_{1} \leq a_{2}+a_{3} .
$$


Moreover $\phi(L)=a$ and $\mu(L)=2 a-2$ or $2 a-1$, so that $F_{1} . L=a$ and $F_{2} . L=a$ or $a+1$. In the first case we have $F_{1} \cdot\left(E_{1}+E_{2}+E_{3}\right)=F_{2} \cdot\left(E_{1}+E_{2}+E_{3}\right)=3$, and from Lemma 2.10 we get $F_{1} \cdot F_{2}=1$, a contradiction. In the second case, using our assumption that $\mu(L)<2 a+a_{3}-2$, we must have $a_{3} \geq 2$, and consequently $a_{2} \geq 2$. Hence $a_{1} \geq 2$ as well by (8). Therefore $F_{2} \cdot L=a_{1} F_{2} \cdot E_{1}+a_{2} F_{2} \cdot E_{2}+a_{3} F_{2} \cdot E_{3}=a$ or $\geq a+2$, a contradiction.

Assume next that $F_{1} \equiv E_{i}$ for some $i$ but $F_{2}$ is not. Then, using (17), we can assume $F_{1} \equiv E_{1}, E_{2}$ or $E_{4}$, after renumbering indices (but still maintaining the inequalities in (6)).

If $F_{1} \equiv E_{1}$ then $F_{1} \cdot L=\phi(L)=a+a_{2}+a_{3}-a_{1}$ and $F_{2} \cdot L \geq a+a_{1}$, whence $\mu(L) \geq$ $2 a+a_{2}+a_{3}-2 \geq 2 a+2 a_{3}-2>2 a+a_{3}-2$, a contradiction.

If $F_{1} \equiv E_{2}$ then $F_{1} . L=\phi(L)=a-a_{2}+a_{1}$ and $F_{2} . L \geq a+a_{2}$, whence $\mu(L) \geq 2 a+a_{1}-2$. From (17) we get

$$
2 a+a_{1}-2 \leq \mu(L)<2 \phi(L)=2\left(a-a_{2}+a_{1}\right),
$$

whence $2 a_{2} \leq a_{1}+1$ and $a-a_{2}+a_{1} \leq a-a_{4}$, so that $a_{4} \leq a_{2}-a_{1} \leq 1-a_{2} \leq 0$. Therefore $a_{1}=a_{2}=a_{3}=1, a_{4}=a_{5}=\ldots=a_{10}=0$ and we get the contradiction $5=2 a+a_{1}-2 \leq \mu(L)<2 a+a_{3}-2=5$.

If $F_{1} \equiv E_{4}$ then $F_{1} \cdot L=\phi(L)=a-a_{4}$ and $a-a_{4}+1=\phi(L)+1 \geq F_{2} . L \geq a+a_{4}$, so that $a_{4}=a_{5}=\ldots=a_{10}=0$ and $\phi(L)=a, \mu(L)=2 a-2$ or $2 a-1$. Moreover, by (7) we see that (8) holds and we derive the same contradiction as above, right after (8).

Now assume that $F_{2} \equiv E_{i}$ for some $i$ but $F_{1}$ is not. Then $a \leq F_{1} . L=\phi(L) \leq a-a_{4}$ whence $a_{4}=\ldots=a_{10}=0$ and $F_{1} \cdot E_{i}=1$ for $1 \leq i \leq 3$. Since $F_{1} \cdot F_{2}=2$ we get $F_{2} \equiv E_{j}$ for some $j \geq 4$, but then $F_{1} \cdot\left(E_{1}+E_{2}+E_{3}\right)=F_{2} \cdot\left(E_{1}+E_{2}+E_{3}\right)=3$, contradicting Lemma 2.10 .

We have therefore proved that if $\mu(L)<2 a+a_{3}-2$ then $F_{1} \equiv E_{i}, F_{2} \equiv E_{j}$ for some $i, j$. By (6) and (7) we can assume $F_{1} \equiv E_{1}$ and $F_{2} \equiv E_{2}, E_{3}$ or $F_{1} \equiv E_{2}$ and $F_{2} \equiv E_{1}$. Now $\left(E_{1}+E_{2}\right) \cdot L=2 a+a_{3} \leq 2 a+a_{2}=\left(E_{1}+E_{3}\right) . L$, contradicting $\mu(L)<2 a+a_{3}-2$.

This proves that $\mu(L) \geq 2 a+a_{3}-2$. Comparing with (7) we have

$$
2 a+a_{3}-2 \leq \mu(L)<2 \phi(L)=\min \left\{2\left(a+a_{2}+a_{3}-a_{1}\right), 2\left(a-a_{2}+a_{1}\right), 2\left(a-a_{4}\right)\right\} .
$$

Then $2 a+a_{3}-2<2 a-2 a_{4}$, whence $a_{3}=1, a_{4}=\ldots=a_{10}=0$ and $a_{2} \leq a_{1} \leq a_{2}+1$, so that $L$ is of type $\left(\mu_{2}\right)$ or $\left(\mu_{3}\right)$.

\subsection{A result on the positive cone of an Enriques surface.}

Proof of Proposition 1. We prove the proposition by induction on $L^{2}$. It is easily seen to hold fo $\phi(L) \leq 3$. If $\phi(L)=4$ we have that either $L^{2}=16$ and we get case (i) by Lemma 2.11 or $L^{2}=18$. In the latter case let $E>0$ be such that $E^{2}=0$ and $E . L=4$. Write $L-2 E \sim E_{1}+E_{2}$ for $E_{i}>0$ with $E_{i}^{2}=0, i=1,2$ and $E_{1} . E_{2}=1$ by Lemma 2.4. Since $4=\phi(L) \leq E_{i} . L=2 E \cdot E_{i}+1$, we find $E \cdot E_{i}=2$ for $i=1,2$, whence $L$ is as in case (ii-b).

We will henceforth assume that $\phi(L) \geq 5$ and therefore $L^{2} \geq 26$.

Pick an $E>0$ such that $E^{2}=0$ and $E . L=\phi(L)$. Then $(L-E)^{2}>0$ and $L-E>0$ by Lemma 2.4. Moreover $\phi(L)=E . L=E .(L-E) \geq \phi(L-E)$. If $\phi(L-E)=\phi(L)$, then

$$
(L-E)^{2}=L^{2}-2 \phi(L) \leq \phi(L)^{2}-\phi(L)-2<\phi(L)^{2}=\phi(L-E)^{2},
$$

a contradiction. If $L^{2}=\phi(L)^{2}$ and $\phi(L-E)=\phi(L)-1$, then $(L-E)^{2}=\phi(L-E)^{2}-1$, again a contradiction. We have therefore proved that

$$
\phi(L-E) \leq \phi(L)-1 \text { with equality only if } L^{2}>\phi(L)^{2} .
$$

Now pick an $E^{\prime}>0$ such that $\left(E^{\prime}\right)^{2}=0$ and $E^{\prime} .(L-E)=\phi(L-E)$.

Assume $\phi(L-E) \leq \phi(L)-2$. From $\phi(L) \leq E^{\prime} . L=\phi(L-E)+E$. $E^{\prime}$, we find $E . E^{\prime} \geq 2$. If equality holds, then Proposition 2.8 and Lemma 2.14 imply that $L$ is as in case (i). 
If $3 \leq E . E^{\prime} \leq 6$, then $6 \leq\left(E+E^{\prime}\right)^{2} \leq 12$ and it is easily shown that $E+E^{\prime}$ is a sum of at least three divisors $F_{i}>0$ with $F_{i}^{2}=0$, whence $\left(E+E^{\prime}\right) \cdot L \geq 3 \phi(L)$. Now $E^{\prime} . L \leq \phi(L)-2+E \cdot E^{\prime} \leq \phi(L)+4$, whence $\phi(L) \leq 4$, a contradiction.

If $E . E^{\prime} \geq 7$ we set $\alpha=\left\lfloor\frac{\phi(L)}{2}\right\rfloor-1$. Then $\left(L-E-\alpha E^{\prime}\right)^{2} \geq 2 \phi(L)-4 \geq 6$, so that $L-E-\alpha E^{\prime}>0$ by Lemma 2.4 , whence

$$
\phi(L)=E \cdot L=E \cdot\left(L-E-\alpha E^{\prime}\right)+\alpha E \cdot E^{\prime} \geq 1+7 \alpha \geq 1+7\left(\frac{\phi(L)-3}{2}\right),
$$

giving the contradiction $\phi(L) \leq 3$.

We have therefore proved that one of the following holds:

$$
\begin{aligned}
& \phi(L-E)=\phi(L)-1 \text { and } L^{2}>\phi(L)^{2}, \\
& \phi(L-E)=\phi(L)-2 \text { and } L^{2}=\phi(L)^{2} .
\end{aligned}
$$

Of course in case (11) we get that $L$ is as in (i) by Lemma 2.11.

Now assume that we are in case (10). Then

$$
(L-E)^{2}=L^{2}-2 \phi(L) \leq \phi(L)^{2}-\phi(L)-2=\phi(L-E)^{2}+\phi(L-E)-2 .
$$

If $(L-E)^{2}=\phi(L-E)^{2}$, then, by Lemma 2.11, we have that $L-E \equiv h\left(E_{1}+E_{2}\right)$ with $h \geq 1, E_{i}>0, E_{i}^{2}=0$ and $E_{1} \cdot E_{2}=2$. In particular $\phi(L-E)=2 h$, so that $\phi(L)=2 h+1$. It follows that $h \geq 2$. Now $\phi(L)=2 h+1=E \cdot L=h\left(E . E_{1}+E \cdot E_{2}\right)$, whence we must have either $E . E_{1} \geq 2$ or $E . E_{2} \geq 2$. Moreover $E . E_{i}>0$ for $i=1,2$ by Lemma 2.2 as $E$. $(L-E)>E_{i} .(L-E)$. Hence $\phi(L) \geq 3 h$, a contradiction. Therefore we have

$$
\phi(L-E)^{2}<(L-E)^{2} \leq \phi(L-E)^{2}+\phi(L-E)-2,
$$

and we can assume by induction that in fact $(L-E)^{2}=\phi(L-E)^{2}+\phi(L-E)-2$ (and, consequently, $\left.L^{2}=\phi(L)^{2}+\phi(L)-2\right)$ and that we are in one of the three following cases, where $h \geq 1$, all the $E_{i}$ 's are primitive, $E_{i}>0, E_{i}^{2}=0, E_{1} \cdot E_{2}=E_{1} \cdot E_{3}=2$ and $E_{2} \cdot E_{3}=1$ :

(a) $L-E \sim h\left(E_{1}+E_{2}\right)+E_{3}, \phi(L-E)=2 h+1$,

(b) $L-E \sim(h+1) E_{1}+h E_{2}+E_{3}, \phi(L-E)=2 h+2$,

(c) $L-E \equiv 2\left(E_{1}+E_{2}+E_{3}\right), \phi(L-E)=6$.

Now case (c) cannot occur since we have $E . L=\phi(L)=\phi(L-E)+1=7$.

In case (a) we have $E .(L-E)=\phi(L)=\phi(L-E)+1=2 h+2$, whence $h \geq 2$, $E_{1} \cdot(L-E)=2 h+2, E_{2} \cdot(L-E)=2 h+1$ and $E_{3} \cdot(L-E)=3 h$. Since $E$ and all the $E_{i}$ 's are primitive, we must have $E . E_{2}>0$ by Lemma 2.2. For the same reason, if $E . E_{3}=0$ we must have $E \equiv E_{3}$ and $h=2$. Then $L \equiv 2\left(E_{1}+E_{2}+E_{3}\right)$ and we are in case (ii-c). Again, if $E . E_{1}=0$ we must have $E \equiv E_{1}$, whence $L \equiv(h+1) E_{1}+h E_{2}+E_{3}$ and we are in case (ii-b).

Therefore we can assume $E . E_{i}>0$ for all $i=1,2,3$.

If $E . E_{1} \geq 2$ or $E . E_{2} \geq 2$, then $2 h+2=E . L \geq 3 h+1$, a contradiction. Hence $E . E_{1}=E \cdot E_{2}=1$ and $E \cdot E_{3}=2$. Then $E_{1} \cdot L=2 h+3, E_{2} \cdot L=2 h+2$ and $\mu(L) \leq$ $\left(E_{1}+E_{2}\right) \cdot L-2=4 h+3<2 \phi(L)$ and we are in case (ii-b) by Proposition 2.8 and Lemma 2.14 .

In case (b), working as in case (a), we deduce that $E . E_{1}>0$ and either $E \equiv E_{3}, h=1$ and $L \equiv 2 E_{1}+2 E_{3}+E_{2}$ or $E \equiv E_{2}$ and $L \equiv(h+1)\left(E_{1}+E_{2}\right)+E_{3}$ and we are in case (ii-a). Therefore we can assume $E . E_{i}>0$ for all $i=1,2,3$.

If $E . E_{1} \geq 2$, then $2 h+3=E . L \geq 3 h+3$, a contradiction. If $E . E_{2} \geq 2$, then $2 h+3=$ $E . L \geq 3 h+2$, so that we must have $h=1, E . E_{2}=2$ and E. $E_{1}=E \cdot E_{3}=1$. But then $E_{1} \cdot L=5$ and $E_{3} \cdot L=6$, so that $\mu(L) \leq 9<2 \phi(L)=10$, and we must be in case (ii-a) by Proposition 2.8 and Lemma 2.14 . 
Hence $E \cdot E_{1}=E \cdot E_{2}=1, E_{1} \cdot L=2 h+3$ and $E_{2} \cdot L=2 h+4$, whence $\mu(L) \leq\left(E_{1}+E_{2}\right) \cdot L-$ $2=4 h+5<2 \phi(L)$ and we are in case (ii-a) by Proposition 2.8 and Lemma 2.14 .

Remark 2.15. Proposition 1 improves the result of Hana [Ha, Thm.1.8].

Lemma 2.16. If $L \equiv 2 D$ with $D^{2}=10$ and $\phi(D)=3$, then $\mu(L)=2 \phi(L)=12$.

Proof. Apply Lemmas 2.2, 2.4 and 2.14 and Proposition 2.8.

2.3. A few useful applications. A direct application of Lemmas 2.2 2.4 and Theorem 2.6 yields the results in Lemmas 2.17 2.20, which will be of use to us.

Lemma 2.17. Let $|L|$ be a base-point free complete linear system on an Enriques surface $S$ with $L^{2}=10$ and $\phi(L)=2$. Let $E$ be a nef divisor such that $E^{2}=0$ and $E . L=2$. Then

$$
L \sim 2 E+E_{1}+E_{2}
$$

for $E_{i}>0$ primitive with $E_{i}^{2}=0$ and $E \cdot E_{i}=E_{1} \cdot E_{2}=1, i=1,2$ such that

(i) $\left|E+E_{1}\right|$ is base-component free with two distinct base points,

(ii) $E+E_{2}$ is quasi-nef.

Proof. The existence of the decomposition of $L$ follows from Lemmas 2.4 and 2.2 .

Assume there is a $\Delta>0$ such that $\Delta^{2}=-2$ and $\Delta .\left(E+E_{1}\right)<0$. Then $\Delta \cdot E_{1}<0$ by the nefness of $E$. By Lemma 2.3 there is an $A>0$ primitive such that $A^{2}=0$ and $E_{1} \sim A+k \Delta$, where $k=-\Delta . E_{1} \geq 1$. Since $1=E \cdot E_{1}=E \cdot A+k E . \Delta \geq k E . \Delta$ we get that if $\Delta . E>0$ then $k=\Delta . E=1$, whence $\Delta .\left(E+E_{1}\right)=0$, a contradiction. Therefore $\Delta . E=0$, whence $\Delta \cdot E_{2} \geq k$ by the nefness of $L$ and since $1=E_{2} \cdot E_{1}=E_{2} \cdot A+k E_{2} \cdot \Delta$ we get that $k=E_{2} \cdot \Delta=1$ and $A . E_{2}=0$, so that $A \equiv E_{2}$ by Lemma 2.2 . Hence $E_{1} \equiv E_{2}+\Delta$, with $E_{2} \cdot \Delta=1$. In particular $E+E_{1}$ is quasi-nef. Also, if $E+E_{1}$ is not nef then there is a nodal curve $\Gamma$ such that $E_{1} \equiv E_{2}+\Gamma$, with $E_{2} \cdot \Gamma=1$.

Similarly $E+E_{2}$ is quasi-nef and if there is a nodal curve $\Gamma^{\prime}$ such that $\Gamma^{\prime} .\left(E+E_{2}\right)<0$ then $E_{2} \equiv E_{1}+\Gamma^{\prime}$, with $E_{1} \cdot \Gamma^{\prime}=1$.

Obviously it follows that either $E+E_{1}$ or $E+E_{2}$ is nef. By symmetry, we can assume that $E+E_{1}$ is nef, and by [CD, Prop.3.1.6, Cor.3.1.4 and Thm.4.4.1] the lemma is proved, possibly after adding $K_{S}$ to both $E_{1}$ and $E_{2}$.

Lemma 2.18. Let $|L|$ be a base-point free complete linear system on an Enriques surface $S$ with $L^{2}=10$ and $\phi(L)=3$. Among all $E>0$ satisfying $E^{2}=0$ and $E . L=3$ pick one which is maximal. Then

$$
L \sim E+E_{1}+E_{2}
$$

for $E_{i}>0$ primitive with $E_{i}^{2}=0, i=1,2, E \cdot E_{1}=1, E \cdot E_{2}=E_{1} \cdot E_{2}=2$ such that

(i) $\left|E_{1}+E_{2}\right|$ and $\left|E_{1}+E_{2}+K_{S}\right|$ are base-point free,

(ii) $h^{1}(2 E-L) \leq 1$.

Proof. The existence of the decomposition of $L$ follows from Lemmas 2.4 and2.2, If $E_{1}+E_{2}$ is not nef, it follows that there is a nodal curve $\Gamma$ such that $(E+\Gamma)^{2}=0$ and $(E+\Gamma) \cdot L=3$, contradicting the maximality of $E$. This proves (i). Note that $h^{0}(2 E-L)=0$ and $h^{0}\left(L-2 E+K_{S}\right) \leq 1$ since $L .\left(L-2 E+K_{S}\right)=4<2 \phi(L)$, giving (ii) by Riemann-Roch.

Lemma 2.19. Let $|L|$ be a base-point free complete linear system on an Enriques surface $S$ with $L^{2}=14$ and $\phi(L)=2$ and let $E>0$ be a nef divisor with $E^{2}=0$ and E. $L=2$. Then there exists a decomposition

$$
L \sim 3 E+E_{1}+E_{2}
$$


with $E_{i}>0, E_{i}^{2}=0$ and $E . E_{i}=E_{1} \cdot E_{2}=1, i=1,2$. Moreover $3 E+E_{1}$ is nef and $h^{0}\left(E_{2}+K_{S}\right)=1$.

Proof. We only prove that $h^{0}\left(E_{2}+K_{S}\right)=1$. If $h^{0}\left(E_{2}+K_{S}\right) \geq 2$ then $h^{1}\left(E_{2}+K_{S}\right) \geq 1$ by Riemann-Roch, whence, using Theorem [2.6, there exists a divisor $\Delta>0$ such that $\Delta . E_{2} \leq-2$. By Lemma 2.3 we can write $E_{2}+K_{S} \sim A+k \Delta$ with $A>0, A^{2}=0$ and $k=-E_{2} . \Delta \geq 2$. If $E . \Delta>0$ we get the contradiction $1=E . E_{2} \geq 2$. Therefore $E . \Delta=0$ and similarly $E_{1} . \Delta \leq 0$, contradicting the nefness of $L$.

Lemma 2.20. Let $|L|$ be a base-point free complete linear system on an Enriques surface $S$ with $L^{2}=16$ and $\phi(L)=3$ and let $E>0$ be a nef divisor with $E^{2}=0$ and E.L $=3$. Then there exists a decomposition

$$
L \sim 2 E+E_{1}+E_{2}
$$

for $E_{i}>0$ with $E_{i}^{2}=0, i=1,2, E \cdot E_{1}=E_{1} \cdot E_{2}=2, E \cdot E_{2}=1$ and $2 E+E_{1}$ is nef. Moreover, either $h^{0}\left(2 E+E_{1}-E_{2}\right)=h^{0}\left(2 E+E_{1}-E_{2}+K_{S}\right)=1$ or $E_{1} \equiv 2 F$, for an $F>0$ with $F^{2}=0$.

Proof. The nefness of $2 E+E_{1}$ follows, as in the previous lemmas, by choosing a maximal $E_{2}$. Now note that $\left(2 E+E_{1}-E_{2}\right)^{2}=0$ and $E .\left(2 E+E_{1}-E_{2}\right)=1$, whence $2 E+E_{1}-E_{2}>0$. If $h^{0}\left(2 E+E_{1}-E_{2}\right)>1$ or if $h^{0}\left(2 E+E_{1}-E_{2}+K_{S}\right)>1$, then by Riemann-Roch and Theorem 2.6, there must exist a $\Delta>0$ with $\Delta^{2}=-2$ and $\Delta .\left(2 E+E_{1}-E_{2}\right) \leq-2$. Since $2 E+E_{1}$ is nef, we must have $\Delta . E_{2} \geq 2$. By Lemma 2.3 there is an $A>0$ primitive such that $A^{2}=0$ and $2 E+E_{1}-E_{2} \sim A+k \Delta$, where $k:=-\Delta \cdot\left(2 E+E_{1}-E_{2}\right) \geq 2$. From $4=E_{2} \cdot\left(2 E+E_{1}-E_{2}\right)=E_{2} \cdot A+k E_{2} \cdot \Delta$ we find that $E_{2} \equiv A$ and $k=2$, so that $2 E+E_{1}-E_{2} \equiv E_{2}+2 \Delta$. Hence $E_{1} \equiv 2\left(E_{2}+\Delta-E\right)$.

\section{A COUple of useful Results Using Vector Bundles Methods}

In the present section we will derive two useful results from the well-known vector bundle methods introduced by Lazarsfeld and Tyurin ([GL, La, T] ). The methods will be pushed a little bit forward on an Enriques surface. To this end, recall that if $C$ is a smooth irreducible curve on a smooth irreducible surface $S$ with $h^{1}\left(\mathcal{O}_{S}\right)=0$ and $A$ is a globally generated line bundle on $C$, one can construct ([La, $[\mathrm{CP}, \overline{\mathrm{Par}}]$ ) a vector bundle $\mathcal{E}(C, A)$ of rank $h^{0}(A)$, with $\operatorname{det} \mathcal{E}(C, A)=\mathcal{O}_{S}(C)$ and fitting into an exact sequence

$$
0 \longrightarrow H^{0}(A)^{*} \otimes \mathcal{O}_{S} \longrightarrow \mathcal{E}(C, A) \longrightarrow \mathcal{N}_{C / S} \otimes A^{-1} \longrightarrow 0
$$

We will make use of the following variant of a well-known result in [DM, Kn, GLM]:

Proposition 3.1. Let $|L|$ be a base-point free complete linear system on an Enriques surface $S$ and assume that there is a smooth irreducible curve $C \in|L|$ with a base-point free line bundle $A$ such that $|A|$ is a $g_{k}^{1}$ and $L^{2} \geq \max \{4 k-2,2 k-2+2 \phi(L)\}$. Then either

(a) there is an effective nontrivial decomposition $L \sim N+N^{\prime}$ such that $\left|N^{\prime}\right|$ is basecomponent free with $2\left(N^{\prime}\right)^{2} \leq L . N^{\prime} \leq\left(N^{\prime}\right)^{2}+k \leq 2 k$ and $N_{\mid C}^{\prime} \geq A$. Either $N \geq N^{\prime}$ or $|N|$ is base-component free and $\mathrm{Bs}|N| \subset C$ and $H .\left(N-N^{\prime}\right) \geq 0$ for any ample H. Moreover if $\phi\left(N^{\prime}\right)=1$ and $L . N^{\prime} \geq\left(N^{\prime}\right)^{2}+k-1$, then $\operatorname{Bs}\left|N^{\prime}\right| \cap C \neq \emptyset$, or

(b) $L^{2}=4 k-2$ and for any $\Delta \geq 0$ such that $h^{0}(\mathcal{E}(C, A)(-\Delta))>0$ we can find a line bundle $N \geq \Delta$ and a nodal cycle $R$ so that $L \sim 2 N+R+K_{S}, R^{2}=-2, h^{0}(R)=1$, $h^{0}\left(R+K_{S}\right)=0,\left|N^{\prime}\right|:=\left|N+R+K_{S}\right|$ is base-component free with $\mathrm{Bs}\left|N^{\prime}\right| \subset C$, $N .(N+R)=k$ and $\left(N+R+K_{S}\right)_{\mid C} \geq A$. 
Proof. Let $E>0$ be any divisor such that $E^{2}=0$ and $E . L=\phi(L)$. Then $h^{0}\left(\mathcal{N}_{C / S}-A-\right.$ $\left.E_{\mid C}\right)=h^{0}\left(\omega_{C}-A-\left(E+K_{S}\right)_{\mid C}\right) \geq h^{1}(A)-\phi(L)=2+\frac{1}{2} L^{2}-k-\phi(L)>0$ by hypothesis. In particular $h^{0}\left(\mathcal{N}_{C / S}-A\right)>0$ so that $\mathcal{E}:=\mathcal{E}(C, A)$ is globally generated off a finite set. Also we have $h^{2}\left(\mathcal{E} \otimes \omega_{S}\right)=0$ and $c_{1}(\mathcal{E})^{2}-4 c_{2}(\mathcal{E}) \geq-2$, whence, as in [DM, Kn, GLM], there exist two line bundles $N, N^{\prime}$ on $S$ and $Z \subset S$ with $\operatorname{dim} Z=0$, such that

$$
0 \longrightarrow N \longrightarrow \mathcal{E} \longrightarrow \mathcal{J}_{Z / S} \otimes N^{\prime} \longrightarrow 0
$$

with $L \sim N+N^{\prime}$ and $k=N \cdot N^{\prime}+\operatorname{length}(Z)$.

Now case (a) corresponds to the case $c_{1}(\mathcal{E})^{2}-4 c_{2}(\mathcal{E}) \geq 0$, using (13) and (14). Suppose that $\phi\left(N^{\prime}\right)=1$ and $L . N^{\prime} \geq\left(N^{\prime}\right)^{2}+k-1$. Then $\left|N^{\prime}\right|$ has two base points by [CD, Thm.4.4.1]. Since $N^{\prime}$ is a quotient of $\mathcal{E}$ off $Z$ and $\mathcal{E}$ is globally generated outside a finite set contained in $C$, the two base points of $\left|N^{\prime}\right|$ must lie on $C \cup Z$. But length $(Z)=\left(N^{\prime}\right)^{2}+k-L . N^{\prime} \leq 1$, whence Bs $\left|N^{\prime}\right| \cap C \neq \emptyset$, as stated. The rest of (a) is proved similarly to [DM], [Kn, §3], GLM, Lemma2.1]. Case (b) corresponds to the case $c_{1}(\mathcal{E})^{2}-4 c_{2}(\mathcal{E})=-2$ and is proved as in [Kn, §3] using [Ki, Thm.3.4].

We will also need the following simple result.

Lemma 3.2. Let $C$ be a smooth irreducible curve on an Enriques surface $S$ and let $A$ be a base-point free line bundle on $C$ with $h^{0}(A)=2$ and $\operatorname{deg} A=k$. Assume that there are two line bundles $M>0, N>0$ such that $C+K_{S} \sim M+N, h^{1}\left(M+K_{S}\right)=0$ and $\frac{1}{2}\left(M^{2}+N^{2}\right)+2>k$. Then either $h^{0}(\mathcal{E}(C, A)(-M))>0$ and $\left(N+K_{S}\right)_{\mid C} \geq A$ or $h^{0}(\mathcal{E}(C, A)(-N))>0$ and $\left(M+K_{S}\right)_{\mid C} \geq A$.

Proof. Set $\mathcal{E}=\mathcal{E}(C, A)$. If $h^{0}\left(\mathcal{O}_{C}\left(N+K_{S}\right)(-A)\right)=0$ we get, using Serre duality, the dual of (13) tensored with $\mathcal{O}_{S}(M)$, Riemann-Roch and the hypotheses, that

$$
\begin{aligned}
h^{0}(\mathcal{E}(-N)) & \geq h^{0}(A) \cdot h^{0}\left(M+K_{S}\right)-h^{0}\left(\mathcal{O}_{C}\left(M+K_{S}\right)(A)\right)= \\
& =2 \chi\left(M+K_{S}\right)-\chi\left(\mathcal{O}_{C}\left(M+K_{S}\right)(A)\right)=\frac{1}{2}\left(M^{2}+N^{2}\right)+2-k>0 .
\end{aligned}
$$

Similarly, if $h^{0}\left(\mathcal{O}_{C}\left(N+K_{S}\right)(-A)\right)>0$, we find that $h^{0}(\mathcal{E}(-M))>0$. To conclude just tensor (13) by $\mathcal{O}_{S}(-M)$ or $\mathcal{O}_{S}(-N)$.

\section{A FramewORK FOR THE STUDY OF GENERIC GONALITY}

The goal of this section will be to devise a method to study the gonality of general curves $C$ in a given complete linear system $|L|$ on a surface. While all previous means of investigation are essentially based on the vector bundle method of Bogomolov, Lazarsfeld, Tyurin and others, and therefore work when instability conditions hold, requiring $L^{2}$ to be large enough, our approach will be, in many cases, independent of such conditions. The idea will be a sort of "liaison" using the zero-dimensional schemes defining the gonality.

We will often use the ensuing two definitions.

Definition 4.1. Let $S$ be an Enriques surface, let $|L|$ be a base-point free complete linear system on $S,|L|_{\text {sm }}$ the open subset of smooth curves in $|L|$ and let $k \geq 3$ be an integer. We will say that a nonempty open subset $\mathcal{U} \subset|L|_{\text {sm }}$ has generic gonality $k$ if the following two conditions hold:

(i) $\operatorname{gon}(C)=k$ for all $C \in \mathcal{U}$;

(ii) if $k=2 \phi(L)$ then $k^{2}<2 L^{2}$ and every $C \in \mathcal{U}$ carries (at least) one $g_{k}^{1}$, $A_{C}$, that is not cut out by a genus one pencil on $S$. 
Definition 4.2. Let $(L, k)$ be as in Definition 4.1 and let $\mathcal{U} \subset|L|_{s m}$ be a nonempty open subset with generic gonality $k$. For every $C \in \mathcal{U}$ let $A_{C}$ be a $g_{k}^{1}$ on $C$ such that, if $k=2 \phi(L)$, $A_{C}$ is not cut out by a genus one pencil on $S$. Let $\left\{D_{1}, \ldots, D_{n}\right\}$ be a set of divisors on $S$.

We will say that $\left\{D_{1}, \ldots, D_{n}\right\}$ is a $\left(\mathcal{U}, L, k,\left\{A_{C}\right\}_{C \in \mathcal{U}}\right)$-dominant set of divisors if for every $C \in \mathcal{U}$ there is an $i \in\{1, \ldots, n\}$ such that $\left|D_{i}\right|$ is base-component free, $L-D_{i}>0$, $h^{1}\left(D_{i}-L\right)=0$ and $\left(D_{i}\right)_{\mid C} \geq A_{C}$.

Let us outline our strategy to study the generic gonality of curves in a complete linear system $|L|$. To prove that a general curve $C \in|L|$ has gonality $k+1$, assume that there is a nonempty open subset $\mathcal{U} \subset|L|_{s m}$ of generic gonality $k$. Make a choice of a $g_{k}^{1}$ for every $C \in \mathcal{U}$ and suppose to have found a $\left(\mathcal{U}, L, k,\left\{A_{C}\right\}_{C \in \mathcal{U}}\right)$-dominant set of divisors $\left\{D_{1}, \ldots, D_{n}\right\}$. Intersecting them with $C$ we find some "unexpected" subschemes $Z_{h}$ and this will imply the existence of a line bundle $M$ that will cut out, outside of $Z_{h}$, the given $g_{k}^{1}$ on every curve $C \in \mathcal{U}$. This will lead to some inequalities on $L^{2}, k, L . M$ etc. (Proposition 4.6) that will be used either to deduce a contradiction or to prove that the gonality is computed by $\mu(L)$.

The crux of the argument will be the following construction, with the help of the $Z_{h}$ 's, of a suitable incidence correspondence.

Let $(L, k)$ be as in Definition 4.1 and let $\mathcal{U} \subset|L|_{s m}$ be a nonempty open subset with generic gonality $k$. Let $M \geq 0$ be an effective line bundle on $S$ with $M^{2} \geq 4$. Define

$$
h_{M}=M . L-k,
$$

and, for every zero-dimensional subscheme $X \subset S$ of length $\frac{1}{2} M^{2}-1$, define

$$
W(X)=\operatorname{Bs}\left|\mathcal{J}_{X / S} \otimes M\right|
$$

and the following incidence subvariety of $\operatorname{Hilb}^{\frac{1}{2} M^{2}-1}(S) \times|L|$ :

$\mathfrak{J}_{M}=\left\{(X, C): C \in \mathcal{U}, X \subset C, \operatorname{dim} W(X)=0, \operatorname{deg}(W(X) \cap C)=h_{M}, h^{0}\left(\mathcal{J}_{X / S} \otimes M\right)=2\right\}$

(where $W(X) \cap C$ denotes the scheme-theoretic intersection), together with its two projections $\pi_{1, M}: \mathfrak{J}_{M} \rightarrow \operatorname{Hilb}^{\frac{1}{2} M^{2}-1}(S)$ and $\pi_{2, M}: \mathfrak{J}_{M} \rightarrow|L|$.

With these in mind we have

Lemma 4.3. Let $(L, k)$ be as in Definition 4.1 and with $L^{2} \geq 8$. Let $\mathcal{U} \subset|L|_{\text {sm }}$ be a nonempty open subset with generic gonality $k$ and let $\left\{D_{1}, \ldots, D_{n}\right\}$ be a $\left(\mathcal{U}, L, k,\left\{A_{C}\right\}_{C \in \mathcal{U}}\right)$ dominant set of divisors. Then there exists a nonempty subset $\left\{D_{i_{1}}, \ldots, D_{i_{s}}\right\}$ of $\left\{D_{1}, \ldots, D_{n}\right\}$ and, for every $j=1, \ldots, s$, a base-point free line bundle $M_{i_{j}}$ on $S$ such that:

(i) $0 \leq M_{i_{j}} \leq D_{i_{j}}$;

(ii) $4 \leq M_{i_{j}}^{2} \leq D_{i_{j}}^{2}$ and $M_{i_{j}}=D_{i_{j}}$ if $M_{i_{j}}^{2}=D_{i_{j}}^{2}$;

(iii) $M_{i_{j}} . L \leq k+M_{i_{j}}^{2}$.

(iv) $\phi\left(D_{i_{j}}\right) \geq \phi\left(M_{i_{j}}\right) \geq 2$.

Furthermore, $\pi_{2, M_{i}}$ is a dominant morphism for at least one $j$.

Proof. Given $C \in \mathcal{U}$ we know by Definition 4.2 that there is an $i \in\{1, \ldots, n\}$ such that $\left|D_{i}\right|$ is base-component free, $L-D_{i}>0, h^{1}\left(D_{i}-L\right)=0$ and $\left(D_{i}\right)_{\mid C} \geq A_{C}$. Pick $Z \in\left|A_{C}\right|$. Then $h^{0}\left(\mathcal{J}_{Z / S} \otimes D_{i}\right)=h^{0}\left(\left(D_{i}\right)_{\mid C}-A_{C}\right) \geq 1$, whence there is a divisor $D_{i, Z} \in\left|\mathcal{J}_{Z / S} \otimes D_{i}\right|$ and we can write $D_{i, Z} \cap C=Z+T\left(C, Z, D_{i, Z}\right)$ as divisors on $C$. We record for later use that $\operatorname{deg}\left(T\left(C, Z, D_{i, Z}\right)\right)=D_{i} . L-k$. Set, for simplicity, $T_{i}=T\left(C, Z, D_{i, Z}\right)$. Then

$$
0 \longrightarrow D_{i}-L \longrightarrow \mathcal{J}_{T_{i} / S} \otimes D_{i} \longrightarrow A_{C} \longrightarrow 0
$$


gives that $h^{0}\left(\mathcal{J}_{T_{i} / S} \otimes D_{i}\right)=2$. Defining $B_{i}=B_{Z, C, D_{i, Z}}$ to be the (possibly empty) basedivisor of $\left|\mathcal{J}_{T_{i} / S} \otimes D_{i}\right|$ and $M_{i}=M_{Z, C, D_{i, Z}}:=D_{i}-B_{i}$, we can write

$$
\left|\mathcal{J}_{T_{i} / S} \otimes D_{i}\right|=\left|V_{i}\right|+B_{i}
$$

where $V_{i}=V_{Z, C, D_{i, Z}} \subseteq H^{0}\left(M_{i}\right)$ is a pencil without base components. In particular $\operatorname{dim}\left|M_{i}\right| \geq 1$ and $\left|M_{i}\right|$ is base-component free. Note also that any such $M_{i}$ satisfies $0 \leq M_{i} \leq D_{i}$. Since there are only finitely many ways of decomposing $D_{i}$ into the sum of two effective divisor classes, we see that, in any case, the possible line bundles $M_{i}=M_{Z, C, D_{i, Z}}$ are finitely many.

Sublemma 4.4. For any $C \in \mathcal{U}, Z \in\left|A_{C}\right|, D_{i, Z} \in\left|\mathcal{J}_{Z / S} \otimes D_{i}\right|$ fix $B_{i}=B_{Z, C, D_{i, Z}}, M_{i}=$ $M_{Z, C, D_{i, Z}}$ and $V_{i}=V_{Z, C, D_{i, Z}}$ as above. Then there are two divisors $T_{M_{i}}=T_{M_{i}}\left(C, Z, D_{i, Z}\right)$, $T_{B_{i}}=T_{B_{i}}\left(C, Z, D_{i, Z}\right)$ giving an effective decomposition $T_{i}=T_{M_{i}}+T_{B_{i}}$ such that

$$
\left|\mathcal{J}_{T_{i} / S} \otimes D_{i}\right|=\left|\mathcal{J}_{T_{M_{i}} / S} \otimes M_{i}\right|+B_{i}, H^{0}\left(\mathcal{J}_{T_{M_{i}} / S} \otimes M_{i}\right)=V_{i}
$$

and with $T_{B_{i}}=C \cap B_{i}, M_{i \mid C} \sim A_{C}+T_{M_{i}}, h_{M_{i}}=\operatorname{deg} T_{M_{i}} \leq M_{i}^{2}$. Moreover (ii) of Lemma 4.3 holds and, possibly after shrinking $\mathcal{U}$ to some nonempty open subset $\mathcal{U}^{\prime}$, we can assume that no $C \in \mathcal{U}^{\prime}$ passes through any of the two base points of all the possible $\left|M_{j}\right|$ that have $\phi\left(M_{j}\right)=1$.

Proof. For simplicity set $B=B_{i}, M=M_{i}, D=D_{i}, T=T_{i}, V=V_{i}$. If $B=0$ we just choose $T_{M}=T$ and $T_{B}=0$. Suppose now that $B>0$. Set $T_{M}:=\operatorname{Bs}\left|V_{\mid C}\right|$. Then $T_{M} \leq T$ as $A_{C}$ is base-point free. Set $T_{B}:=T-T_{M}$. Then one easily sees that $T_{B} \leq C \cap B$. By definition of $T_{M}$ we have an inclusion $V \subseteq H^{0}\left(\mathcal{J}_{T_{M} / S} \otimes M\right)$ and $h^{0}\left(\mathcal{J}_{T_{M} / S} \otimes M\right) \geq 2$. Therefore $h^{0}\left(M_{\mid C}-T_{M}\right) \geq 2$. But $\operatorname{deg}\left(M_{\mid C}-T_{M}\right) \leq k$, whence $T_{B}=C \cap B$, as stated, using the assumption gon $(C)=k$. Now $B_{\mid C}+M_{\mid C} \sim A_{C}+T_{M}+T_{B}$ and it follows that $M_{\mid C} \sim A_{C}+T_{M}$ and $\operatorname{deg} T_{M}=M . L-k=h_{M}$. This implies that $h^{0}\left(M_{\mid C}-T_{M}\right)=h^{0}\left(\mathcal{J}_{T_{M} / S} \otimes M\right)=2$, whence $V=H^{0}\left(\mathcal{J}_{T_{M} / S} \otimes M\right)$ and, by (17), $\left|\mathcal{J}_{T / S} \otimes D\right|=\left|\mathcal{J}_{T_{M} / S} \otimes M\right|+B$.

Since there are only finitely many $M$ 's, we can shrink $\mathcal{U}$ to a nonempty open subset $\mathcal{U}^{\prime}$ such that no $C \in \mathcal{U}^{\prime}$ passes through any of the two base points of all the possible $\left|M_{j}\right|$ that have $\phi\left(M_{j}\right)=1$.

Now two general distinct elements $M^{\prime}$ and $M^{\prime \prime}$ in $|V|=\left|\mathcal{J}_{T_{M} / S} \otimes M\right|$ have no common components, so that $W:=M^{\prime} \cap M^{\prime \prime}$ has dimension zero and degree $M^{2}$. As $T_{M} \subseteq W$ we deduce that $\operatorname{deg} T_{M} \leq M^{2}$. If $M^{2}=0$ we get that $M_{\mid C} \sim A_{C}$, contradicting (ii) of Definition 4.1.

If $M^{2}=2$ then $\left|\mathcal{J}_{T_{M} / S} \otimes M\right|=|M|$ and $|M|$ has two distinct base points $x$ and $y$. Now $T_{M} \subseteq W \cap C=\operatorname{Bs}|M| \cap C=\emptyset$. Therefore $T_{M}=0$ and $M_{\mid C} \sim A_{C}$, whence $k=M . C \geq 2 \phi(L)$ (since $h^{0}(M)=2$ ). As $\operatorname{gon}(C)=k$ we also have $k \leq 2 \phi(L)$, therefore we must have $k=2 \phi(L)$, whence, by (ii) of Definition 4.1. $(M . L)^{2}=k^{2}<2 L^{2}=M^{2} L^{2}$, which contradicts the Hodge index theorem.

Therefore $M^{2} \geq 4$. Now, as $D$ and $M$ are nef, we get $D^{2}=D \cdot(M+B) \geq D \cdot M=$ $(M+B) \cdot M \geq M^{2}$. If equality holds, then $H^{0}(M)=H^{0}(D)$ by Riemann-Roch, so that $B$ is a base-component of $|D|$, whence $B=0$ and (ii) of Lemma 4.3 is proved.

Continuation of the proof of Lemma 4.3. Let $M_{i}$ and $D_{i}$ be the line bundles arising in Sublemma 4.4, If, on any $C \in \mathcal{U}^{\prime}$, we choose a divisor $Z \in\left|A_{C}\right|$ and a divisor $D_{i, Z} \in$ $\left|\mathcal{J}_{Z / S} \otimes D_{i}\right|$, it is easily seen that we can find a divisor $X_{i} \leq T_{M_{i}}$ such that

$$
2=h^{0}\left(\mathcal{J}_{T_{M_{i}} / S} \otimes M_{i}\right)=h^{0}\left(\mathcal{J}_{X_{i} / S} \otimes M_{i}\right)=h^{0}\left(M_{i}\right)-\operatorname{deg} X_{i}=\frac{1}{2} M_{i}^{2}+1-\operatorname{deg} X_{i},
$$


therefore with $h^{0}\left(\mathcal{J}_{X_{i} / S} \otimes M_{i}\right)=2$ and $\operatorname{deg} X_{i}=\frac{1}{2} M_{i}^{2}-1$. Hence $X_{i} \in \operatorname{Hilb}^{\frac{1}{2} M_{i}^{2}-1}(S)$ and, as in (15), $W\left(X_{i}\right)=\mathrm{Bs}\left|\mathcal{J}_{X_{i} / S} \otimes M_{i}\right|$ is such that $\operatorname{dim} W\left(X_{i}\right)=0, \operatorname{length}\left(W\left(X_{i}\right)\right)=M_{i}^{2}$ and $W\left(X_{i}\right)=M_{i}^{\prime} \cap M_{i}^{\prime \prime}$ for two general divisors $M_{i}^{\prime}, M_{i}^{\prime \prime} \in\left|\mathcal{J}_{X_{i} / S} \otimes M_{i}\right|$.

Note that also $T_{M_{i}} \subseteq W\left(X_{i}\right) \cap C$, the scheme-theoretic intersection. In fact we have

Claim 4.5. $T_{M_{i}}=W\left(X_{i}\right) \cap C$ and $\phi\left(D_{i}\right) \geq \phi\left(M_{i}\right) \geq 2$.

Proof. Set $Z^{\prime}=W\left(X_{i}\right) \cap C$ and suppose that $\operatorname{deg} Z^{\prime}>\operatorname{deg} T_{M_{i}}$. Then $h^{0}\left(M_{i \mid C}-Z^{\prime}\right) \geq$ $h^{0}\left(\mathcal{J}_{Z^{\prime} / S} \otimes M_{i}\right) \geq h^{0}\left(\mathcal{J}_{W\left(X_{i}\right) / S} \otimes M_{i}\right)=2$. On the other hand $\operatorname{deg}\left(M_{i \mid C}-Z^{\prime}\right)<M_{i} . L-h_{M_{i}}=$ $k$, contradicting our assumption that $\operatorname{gon}(C)=k$.

To see the second assertion note that, since $D_{i}$ is nef, there is a nef divisor $E$ with $E \cdot D_{i}=$ $\phi\left(D_{i}\right)$, whence $\phi\left(D_{i}\right) \geq \phi\left(M_{i}\right)$. Now assume that $\phi\left(M_{i}\right)=1$. Then $W\left(X_{i}\right)$ contains the two base points of $\left|M_{i}\right|$. Since $W\left(X_{i}\right) \cap C=T_{M_{i}}$ and, by Sublemma 4.4, $C$ does not contain any of them, we must have $h_{M_{i}}=M_{i} . L-k=\operatorname{deg} T_{M_{i}} \leq \operatorname{length}\left(W\left(X_{i}\right)\right)-2 \leq M_{i}^{2}-2$. By Lemma 2.4 we can write $M_{i} \sim \frac{1}{2} M_{i}^{2} E_{1}+E_{2}$ for $E_{p}>0$ with $E_{p}^{2}=0, p=1,2$ and $E_{1} \cdot E_{2}=1$. Hence $\left(\frac{1}{2} M_{i}^{2}+1\right) \phi(L) \leq M_{i} . L \leq k+M_{i}^{2}-2 \leq 2 \phi(L)+M_{i}^{2}-2$. Since $\phi(L) \geq 2$, we deduce that $\phi(L)=2, k=2 \phi(L)=4$ and $E_{1} \cdot L=E_{2} . L=2$. Let $b:=\left\lfloor\frac{L^{2}}{4}\right\rfloor$. Then $\left(L-b E_{1}\right)^{2}=L^{2}-4 b \geq 0$, therefore $L-b E_{1}>0$ by Lemma 2.4 and $2-b=E_{2} .\left(L-b E_{1}\right) \geq 0$, by Lemma 2.2 . Since $L^{2} \geq 8$ by hypothesis we deduce that $b=2$ and $E_{2}$. $\left(L-2 E_{1}\right)=0$ whence, again by Lemma 2.2 , we have that $\left(L-2 E_{1}\right)^{2}=0$, giving $L^{2}=8$. Therefore $k=2 \phi(L)$ and $k^{2}=2 L^{2}$, contradicting (ii) of Definition 4.1

Conclusion of the proof of Lemma 4.3. We have therefore found a nonempty open subset $\mathcal{U}^{\prime} \subset|L|_{s m}$ such that, for any $C \in \mathcal{U}^{\prime}$, by choosing $Z \in\left|A_{C}\right|$ and $D_{i, Z} \in\left|\mathcal{J}_{Z / S} \otimes D_{i}\right|$, we have a line bundle $M_{i}$ satisfying (i), (ii), (iii) and (iv) of Lemma 4.3 (and base-point free by [CD, Prop.3.1.4 and Thm.4.4.1]) and an element $X_{i} \in \operatorname{Hilb}^{\frac{1}{2} M_{i}^{2}-1}(S)$ such that

$$
X_{i} \subset C, \operatorname{dim} W\left(X_{i}\right)=0, \operatorname{deg}\left(W\left(X_{i}\right) \cap C\right)=h_{M_{i}} \text { and } h^{0}\left(\mathcal{J}_{X_{i} / S} \otimes M_{i}\right)=2
$$

whence $\left(X_{i}, C\right) \in \mathfrak{J}_{M_{i}}$, where $\mathfrak{J}_{M_{i}}$ is as in (16). Now if $\left\{i_{1}, \ldots, i_{s}\right\}=\{i: 1 \leq i \leq$ $n$ and there exists $C \in \mathcal{U}^{\prime}$ such that $\left.\left(D_{i}\right)_{\mid C} \geq A_{C}\right\}$, we have proved that $\mathcal{U}^{\prime}=\bigcup_{j=1}^{s} \operatorname{Im} \pi_{2, M_{i}}$. Hence there must be at least one $M_{i_{j}}$ such that $\pi_{2, M_{i}}$ is a dominant morphism.

Proposition 4.6. Let $S$ be an Enriques surface and let $|L|$ be a base-point free complete linear system on $S$ with $L^{2} \geq 8$ and let $k \geq 3$ be an integer. Let $\mathcal{U} \subset|L|_{\text {sm }}$ be a nonempty open subset with generic gonality $k$ and let $\left\{D_{1}, \ldots, D_{n}\right\}$ be a $\left(\mathcal{U}, L, k,\left\{A_{C}\right\}_{C \in \mathcal{U}}\right)$-dominant set of divisors. Then there exists a base-point free line bundle $M$ on $S$ such that:

(a) For at least one $i$ we have $M \leq D_{i},\left|D_{i}\right|$ is base-component free, $L-D_{i}>0, h^{1}\left(D_{i}-L\right)=$ $0, \phi\left(D_{i}\right) \geq \phi(M) \geq 2,4 \leq M^{2} \leq D_{i}^{2}$ and $M=D_{i}$ if $M^{2}=D_{i}^{2}$. Moreover M.L $\leq k+M^{2}$.

(b) If $h_{M}:=M \cdot L-k \geq M^{2}-1$ we have

$$
3 M . L \leq 3 M^{2}+\frac{1}{2} L^{2}+2 h^{1}(L-M)+h^{1}(L-2 M)+k-1
$$

and

$$
M^{2}-M \cdot L+k+2 h^{1}(L-M)+h^{0}\left(2 M-L+K_{S}\right) \geq 2 .
$$

(c) If equality occurs in (18) then

$$
M . L=M^{2}+k \text { and } h^{0}(L-2 M)=0 .
$$


Proof. Let $M=M_{i}$ be a line bundle arising from Lemma 4.3 for which $\pi_{2, M_{i}}$ is a dominant morphism and set $D=D_{i}$. Then (a) follows from Lemma 4.3, To see (b) we study the fibers of the map $\pi_{1, M}$ of $\mathfrak{J}_{M}$ in (16). For $X \in \operatorname{Im} \pi_{1, M}$ consider the set

$$
\Sigma_{X}=\{Y \subset S: Y=W(X) \cap C \text { for some } C \in \mathcal{U}\},
$$

where $W(X) \cap C$ denotes the scheme-theoretic intersection. By definition of $\mathfrak{J}_{M}$ we have, for any $Y \in \Sigma_{X}$, that $\operatorname{deg} Y=\operatorname{deg}(W(X) \cap C)=h_{M} \geq M^{2}-1$, while $Y \subseteq W(X)$ and length $(W(X))=M^{2}$. Therefore either $h_{M}=M^{2}$ and $Y=W(X)$ or $h_{M}=M^{2}-1$ and $Y$ is a maximal subscheme of $W(X)$. Now $W(X)=\operatorname{Bs}\left|\mathcal{J}_{X / S} \otimes M\right|$ and $h^{0}\left(\mathcal{J}_{X / S} \otimes M\right)=2$, whence $W(X)$ is a locally complete intersection subscheme of $S$, therefore it is Gorenstein, whence it has finitely many maximal subschemes. Therefore, in either case, we deduce that $\Sigma_{X}=\left\{Y_{1}, \ldots, Y_{t}\right\}$. Now if $(X, C) \in\left(\pi_{1, M}\right)^{-1}(X)$ then $W(X) \cap C=Y_{j}$ for some $j=1, \ldots, t$. Therefore we have the following inclusion in $|L|$

$$
\left(\pi_{1, M}\right)^{-1}(X) \subseteq \bigcup_{j=1}^{t}\left|H^{0}\left(\mathcal{J}_{Y_{j} / S} \otimes L\right)\right|
$$

whence

$$
\operatorname{dim}\left(\pi_{1, M}\right)^{-1}(X) \leq \max \left\{h^{0}\left(\mathcal{J}_{Y / S} \otimes L\right)-1, Y \in \Sigma_{X}\right\} .
$$

To estimate $h^{0}\left(\mathcal{J}_{Y / S} \otimes L\right)$ we will use the exact sequence

$$
0 \longrightarrow \mathcal{J}_{W(X) / S} \otimes L \longrightarrow \mathcal{J}_{Y / S} \otimes L \longrightarrow \mathcal{J}_{Y / W(X)} \otimes L \longrightarrow 0,
$$

and

$$
h^{0}\left(\mathcal{J}_{Y / W(X)} \otimes L\right)=\chi\left(\mathcal{J}_{Y / W(X)} \otimes L\right)=\chi\left(\mathcal{O}_{W(X)} \otimes L\right)-\chi\left(\mathcal{O}_{Y} \otimes L\right)=M^{2}-h_{M} .
$$

Since $W(X)=M^{\prime} \cap M^{\prime \prime}$ for two distinct $M^{\prime}, M^{\prime \prime} \in\left|\mathcal{J}_{X / S} \otimes M\right|$, we have

$$
0 \longrightarrow L-M \longrightarrow \mathcal{J}_{W(X) / S} \otimes L \longrightarrow \mathcal{J}_{W(X) / M^{\prime}} \otimes L \longrightarrow 0
$$

whence

$$
\begin{aligned}
h^{0}\left(\mathcal{J}_{W(X) / S} \otimes L\right) & \leq h^{0}(L-M)+h^{0}\left(\mathcal{J}_{W(X) / M^{\prime}} \otimes L\right)=h^{0}(L-M)+h^{0}\left(\mathcal{O}_{M^{\prime}}(L-M)\right) \leq \\
& \leq 2 h^{0}(L-M)+h^{1}(L-2 M) .
\end{aligned}
$$

Combining the latter inequality with (21) and (22) we find

$$
\operatorname{dim}\left(\pi_{1, M}\right)^{-1}(X) \leq M^{2}-h_{M}+2 h^{0}(L-M)+h^{1}(L-2 M)-1 .
$$

Note now that the latter bound does not depend any more on $X$. Hence, using the fact that $\operatorname{dim} \operatorname{Hilb}^{\frac{1}{2} M^{2}-1}(S)=M^{2}-2$, we have, for any irreducible component $\mathfrak{J}$ of $\mathfrak{J}_{M}$, that

$$
\operatorname{dim} \mathfrak{J} \leq 2 M^{2}-3+2 h^{0}(L-M)+h^{1}(L-2 M)-h_{M} .
$$

Since $\pi_{2, M}$ is a dominant morphism, there must be a component $\mathfrak{J}_{0}$ of $\mathfrak{J}_{M}$ such that $\operatorname{dim} \mathfrak{J}_{0} \geq \operatorname{dim}|L|=\frac{1}{2} L^{2}$, whence

$$
\frac{1}{2} L^{2} \leq 2 M^{2}-3+2 h^{0}(L-M)+h^{1}(L-2 M)-M . L+k .
$$

Since $h^{2}(L-M) \leq h^{2}(L-D)=0$, we obtain (18) combining Riemann-Roch and (24).

On the other hand the inclusions $Y \subseteq W(X)=M^{\prime} \cap M^{\prime \prime} \subset M^{\prime}$ give the exact sequences

$$
\begin{gathered}
0 \longrightarrow L-M \longrightarrow \mathcal{J}_{Y / S} \otimes L \longrightarrow \mathcal{J}_{Y / M^{\prime}} \otimes L \longrightarrow 0, \\
0 \longrightarrow \mathcal{O}_{M^{\prime}}(L-M) \longrightarrow \mathcal{J}_{Y / M^{\prime}} \otimes L \longrightarrow \mathcal{J}_{Y / W(X)} \otimes L \longrightarrow 0 .
\end{gathered}
$$


Therefore

$$
h^{1}\left(\mathcal{J}_{Y / M^{\prime}} \otimes L\right) \leq h^{1}\left(\mathcal{O}_{M^{\prime}}(L-M)\right) \leq h^{1}(L-M)+h^{2}(L-2 M)
$$

and

$$
h^{1}\left(\mathcal{J}_{Y / S} \otimes L\right) \leq h^{1}(L-M)+h^{1}\left(\mathcal{J}_{Y / M^{\prime}} \otimes L\right) \leq 2 h^{1}(L-M)+h^{2}(L-2 M),
$$

whence

$$
h^{0}\left(\mathcal{J}_{Y / S} \otimes L\right) \leq h^{0}(L)-h_{M}+2 h^{1}(L-M)+h^{2}(L-2 M),
$$

so that, as above,

$$
\operatorname{dim} \mathfrak{J} \leq \operatorname{dim}|L|+M^{2}-2-h_{M}+2 h^{1}(L-M)+h^{0}\left(2 M-L+K_{S}\right)
$$

for every irreducible component $\mathfrak{J}$ of $\mathfrak{J}_{M}$. Since $\pi_{2, M}$ is a dominant morphism, there must be a component $\mathfrak{J}_{0}$ of $\mathfrak{J}_{M}$ such that $\operatorname{dim} \mathfrak{J}_{0} \geq \operatorname{dim}|L|$, whence (19) follows.

Assume now that equality occurs in (18). Then, by Riemann-Roch, $h^{0}(L-2 M)+h^{0}(2 M-$ $\left.L+K_{S}\right)=h_{M}-M^{2}+2-2 h^{1}(L-M)$. Recalling that $M^{2}-1 \leq h_{M} \leq M^{2}$, we get the following three possibilities, where we set, for simplicity, $\alpha=h^{0}(L-2 M)+h^{0}\left(2 M-L+K_{S}\right)$ and $\beta=h^{1}(L-M)$ :

$$
\left(\alpha, h_{M}, \beta\right)=\left(0, M^{2}, 1\right),\left(2, M^{2}, 0\right),\left(1, M^{2}-1,0\right) .
$$

Now equality must also occur in (23) for $\mathfrak{J}=\mathfrak{J}_{0}$, so that it follows that the restriction of $\pi_{1, M}$ to $\mathfrak{J}_{0}$ is a dominant morphism. We know that there is a nonempty open subset $\mathcal{V} \subset \operatorname{Hilb}^{\frac{1}{2} M^{2}-1}(S)$ of zero-dimensional subschemes that impose independent conditions to $|M|$, whence the incidence correspondence

$$
\mathcal{J}_{M}=\{(X, C): X \in \mathcal{V}, C \in|M|, X \subset C\}
$$

has both projections to $\mathcal{V}$ and to $|M|$ that are dominant morphisms. Therefore a general element $X \in \operatorname{Hilb}^{\frac{1}{2} M^{2}-1}(S)$ lies on a general element $M^{\prime} \in|M|$. In particular we can pick $X \in \operatorname{Hilb}^{\frac{1}{2} M^{2}-1}(S)$ general so that $W(X)=M^{\prime} \cap M^{\prime \prime}$ with $M^{\prime}$ a smooth irreducible curve in $\left|\mathcal{J}_{X / S} \otimes M\right|$. Moreover, in case $h^{0}\left(2 M-L+K_{S}\right)=1$, if we let $\Delta$ be the unique element of $\left|2 M-L+K_{S}\right|$, we can also assume that a general element $X \in \operatorname{Hilb}^{\frac{1}{2}} M^{2}-1(S)$ does not intersect $\Delta$. Now let $\varphi_{M}: S \rightarrow \mathbb{P}^{\frac{1}{2} M^{2}}$ be the morphism defined by $|M|$. Since $X \cap \Delta=\emptyset$ and $\varphi_{M}(S)$ is a surface (since $M^{2} \geq 4$ ), we can further assume that the curve (or point) $\varphi_{M}(\Delta)$ does not intersect a general $\left(\mathbb{P}^{\frac{1}{2} M^{2}-2}\right)$-plane in $\mathbb{P}^{\frac{1}{2} M^{2}}$.

Now, in the case $\left(\alpha, h_{M}, \beta\right)=\left(1, M^{2}-1,0\right)$, there is a point $p \in S$ such that

$$
Y+p=W(X) \text { as divisors on } M^{\prime} \text {. }
$$

We henceforth set $W^{\prime}=W(X)-Y$ on $M^{\prime}$, so that $W^{\prime}=p$ in the case $\left(\alpha, h_{M}, \beta\right)=$ $\left(1, M^{2}-1,0\right)$ and $W^{\prime}=0$ in the two other cases in (26). Moreover, since $M^{\prime}$ is smooth we have $\mathcal{J}_{Y / M^{\prime}} \cong \mathcal{J}_{W(X) / M^{\prime}}\left(W^{\prime}\right) \cong \mathcal{O}_{M^{\prime}}(-M)\left(W^{\prime}\right)$.

We have $M^{2}-h_{M}+2 \beta+\alpha-2=0$ in all the cases in (26) , and since $h^{0}\left(2 M-L+K_{S}\right) \leq \alpha$, we must have by (19) that $\alpha=h^{0}\left(2 M-L+K_{S}\right), h^{0}(L-2 M)=0$ and therefore that equality occurs in (19), whence it must also occur in (25), so that

$$
h^{1}\left(\mathcal{O}_{M^{\prime}}(L-M)\left(W^{\prime}\right)\right)=h^{1}(L-M)+h^{2}(L-2 M)=\beta+\alpha .
$$

In case $\left(\alpha, h_{M}, \beta\right)=\left(1, M^{2}-1,0\right)$ we have $W^{\prime}=p$ and $h^{1}\left(M-L+K_{S}\right)=\beta=0$ and from (27), Serre duality and

$$
0 \longrightarrow M-L+K_{S} \longrightarrow \mathcal{J}_{\{p\} / S} \otimes\left(2 M-L+K_{S}\right) \longrightarrow \mathcal{O}_{M^{\prime}}\left(2 M-L+K_{S}\right)(-p) \longrightarrow 0
$$


we see that

$$
h^{0}\left(\mathcal{J}_{\{p\} / S} \otimes\left(2 M-L+K_{S}\right)\right) \geq h^{0}\left(\mathcal{O}_{M^{\prime}}\left(2 M-L+K_{S}\right)(-p)\right)=h^{1}\left(\mathcal{O}_{M^{\prime}}(L-M)(p)\right)=1
$$

whence $h^{0}\left(2 M-L+K_{S}\right)=h^{0}\left(\mathcal{J}_{\{p\} / S} \otimes\left(2 M-L+K_{S}\right)\right)=1$. This means that the unique element $\Delta$ of $\left|2 M-L+K_{S}\right|$ contains $p$. Since $X \cap \Delta=\emptyset$ we have $p \notin X$, but since $p \in W(X)$ and $H^{0}\left(\mathcal{J}_{W(X) / S} \otimes M\right)=H^{0}\left(\mathcal{J}_{X / S} \otimes M\right)$ we have that $\varphi_{M}(p)$ is contained in the linear span of $\varphi_{M}(X)$, which is a general $\mathbb{P}^{\frac{1}{2} M^{2}-2}$. Since $\varphi_{M}(p) \in \varphi_{M}(\Delta)$, this is a contradiction.

Therefore the third case in (26) does not occur. Hence $h^{0}(L-2 M)=0, M . L=M^{2}-k$ and (20) is proved.

Now we need a simple, auxiliary result:

Lemma 4.7. Let $L$ be a line bundle on an Enriques surface $S$ with $L^{2}>0$ and assume $L \sim M+N$ is a nontrivial, effective decomposition such that $M^{2} \geq 0$. Set $l=M . N$.

(a) If $l \leq 2 \phi(L)-2, M^{2} \geq 4, h^{0}\left(2 M-L+K_{S}\right) \leq 2$ and $L^{2} \geq 4 l+4$, then there is a line bundle $M_{0}>0$ such that $M_{0}^{2}=4$ and $M_{0} \cdot L \leq l+4$.

(b) If $L$ is nef, $N^{2} \geq 0, l \leq 2 \phi(L)-1, L^{2} \geq 4 l-4$ and $h^{1}(M) \neq 0$, then there is a line bundle $M_{0}>0$ such that $M_{0}^{2}=4$ and $M_{0} . L \leq l+2$.

Proof. (a) We have $(L-2 M)^{2}=(M-N)^{2}=L^{2}-4 M . N=L^{2}-4 l \geq 4$ by assumption, whence by Riemann-Roch and our assumptions, we get that $h^{0}(L-2 M) \geq 3$.

If $M^{2}=4$, then $M . L=M^{2}+M \cdot N=l+4$ and we are done.

If $M^{2} \geq 6$, then pick $E>0$ such that $E^{2}=0$ and $E . M=\phi(M)$. Then $(M-E)^{2} \geq 2$ whence $M-E>0$ by Lemma 2.4. Moreover, $(M-E) .(N+E) \leq l-1$, since $E$. $(L-2 M) \geq$ $\phi(L-2 M) \geq 1$. If $(M-E)^{2}=2$ we get $2 \phi(L) \leq(M-E) \cdot L \leq l+1 \leq 2 \phi(L)-1$, a contradiction. Hence $(M-E)^{2} \geq 4$, and since $(M-E)^{2}<M^{2}, h^{0}\left(2(M-E)-L+K_{S}\right) \leq$ $h^{0}\left(2 M-L+K_{S}\right) \leq 2, l-1 \leq 2 \phi(L)-3$ and $L^{2}>4(l-1)+4$, we can repeat the process if necessary, which must eventually end.

(b) If $M^{2}=0$, then $h^{0}(M) \geq 2$ implies $2 \phi(L) \leq M . L=M . N=l \leq 2 \phi(L)-1$, a contradiction. Hence $M^{2}>0$, and by Theorem 2.6 there is a $\Delta>0$ such that $\Delta^{2}=-2$ and $\Delta . M \leq-2$. Set $M^{\prime}=M-\Delta$ and $N^{\prime}=N+\Delta$. Note that $M^{\prime}>0$ by Lemma 2.3. We have $\left(M^{\prime}\right)^{2} \geq 4$ and $M^{\prime} . N^{\prime} \leq l-2 \leq 2 \phi(L)-3$. Since $L^{2} \geq 4 l-4=4(l-2)+4$, we are done by (a) if $h^{0}\left(2 M^{\prime}-L+K_{S}\right) \leq 2$.

Assume therefore that $h^{0}\left(2 M^{\prime}-L+K_{S}\right) \geq 3$. This implies $h^{0}\left(2 N^{\prime}-L+K_{S}\right)=0$. Now $\left(N^{\prime}\right)^{2}=(N+\Delta)^{2} \geq N^{2}+2 \geq 2$ (recall that $\Delta . N \geq-\Delta . M \geq 2$ since $L$ is nef). If $\left(N^{\prime}\right)^{2}=2$ then $2 \phi(L) \leq N^{\prime} \cdot L=\left(N^{\prime}\right)^{2}+N^{\prime} \cdot M^{\prime} \leq l \leq 2 \phi(L)-1$, a contradiction.

Therefore $\left(N^{\prime}\right)^{2} \geq 4$ and we are done again by (a).

This allows us to prove the main result of this section:

Proposition 4.8. Let $S$ be an Enriques surface and let $|L|$ be a base-point free complete linear system on $S$ with $L^{2} \geq 8$ and let $k$ be an integer such that $3 \leq k \leq 2 \phi(L)-1$ and $L^{2} \geq 4 k-4$. Let $\mathcal{U} \subset|L|_{\text {sm }}$ be a nonempty open subset with generic gonality $k$ and let $\left\{D_{1}, \ldots, D_{n}\right\}$ be a $\left(\mathcal{U}, L, k,\left\{A_{C}\right\}_{C \in \mathcal{U}}\right)$-dominant set of divisors such that $\left(L-D_{j}\right)^{2} \geq 0$, $L^{2} \geq D_{j}^{2}+2 k-2$ and $h^{0}\left(2 D_{j}-L+K_{S}\right) \leq 1$ for all $j=1, \ldots, n$. Then there exists a line bundle $M_{0}>0$ such that $M_{0}^{2}=4$ and $M_{0} \cdot L \leq k+2$.

Proof. Let $M$ be a line bundle obtained in Proposition 4.6. Then $L-M \geq L-D_{i}>0$ for some $i, M . L-M^{2} \leq k$ and $4 \leq M^{2} \leq D_{i}^{2}$. Hence $h^{0}\left(2 M-L+K_{S}\right) \leq h^{0}\left(2 D_{i}-L+K_{S}\right) \leq 1$. Since $k-2 \leq 2 \phi(L)-3$, we are done by Lemma 4.7(a) if $M . L-M^{2} \leq k-2$. 
Therefore we can assume M.L $-M^{2} \geq k-1$ and we can apply Proposition 4.6(b).

We now claim that $(L-M)^{2} \geq 0$. Indeed if $M^{2}=D_{i}^{2}$ then $M=D_{i}$ by Proposition 4.6(a), so that $(L-M)^{2}=\left(L-D_{i}\right)^{2} \geq 0$. On the other hand if $M^{2} \leq D_{i}^{2}-2$ then $(L-M)^{2} \geq L^{2}-2 k-D_{i}^{2}+2 \geq 0$. Thus if $h^{1}(L-M) \neq 0$ we are done again by Lemma 4.7(b). Therefore we can also assume $h^{1}(L-M)=0$. From Proposition 4.6(b) we get

$$
2 \leq M^{2}-M . L+k+h^{0}\left(2 M-L+K_{S}\right) \leq h^{0}\left(2 M-L+K_{S}\right)+1,
$$

whence $h^{0}\left(2 M-L+K_{S}\right)=1$. From Riemann-Roch and (18) we get

$$
3 M . L \leq 3 M^{2}+\frac{1}{2} L^{2}+h^{1}(L-2 M)+k-1=2 M . L+M^{2}+k-1,
$$

whence $M . L-M^{2}=k-1$ from our assumptions, so that equality occurs in (18). But this contradicts Proposition 4.6(c).

Let $L$ be a nef line bundle on an Enriques surface $S$ with $L^{2} \geq 8$ and $\phi(L) \geq 2$, so that [CD, Prop.3.1.6, Prop.3.1.4, Prop.4.5.1 and Thm.4.5.4] $|L|$ is base-point free and a general curve $C_{\eta} \in|L|$ is not hyperelliptic. We henceforth set

$$
\Sigma_{s}=\left\{C \in|L|_{s m}: \operatorname{gon}(C) \leq s\right\} .
$$

As is well-known $\Sigma_{s}$ is a closed subset of $|L|_{s m}$.

The following two results, direct applications of Proposition 4.8, will also be key results to prove Theorem 1, In their proofs we will use the following simple

Remark 4.9. Let $L$ be a line bundle on an Enriques surface $S$ such that $L^{2}>0$ and let $b$ be an integer such that $L^{2} \geq 4 b+2$. Then $L^{2} \geq 2 b+2 \phi(L)$.

Lemma 4.10. Let $S$ be an Enriques surface and let $|L|$ be a base-point free complete linear system on $S$. Assume that a general smooth curve in $|L|$ has gonality $k \geq 3$ and that either

(i) $L^{2} \geq 4 k$, or

(ii) $L^{2}=4 k-2$ and there is an effective nontrivial decomposition $L \sim M+N$ such that $M^{2} \geq 0, N^{2} \geq 0$ and $k-1 \leq M . N \leq k$.

Then $k \geq \min \{2 \phi(L), \mu(L)\}$.

Proof. As $|L|$ is base-point free we have $\phi(L) \geq 2$ by [CD, Prop.3.1.6, Prop.3.1.4 and Thm.4.4.1]. We assume $k \leq 2 \phi(L)-1$ and we show the existence of a line bundle $M_{0}>0$ such that $M_{0}^{2}=4$ and $M_{0} . L \leq k+2$. This will be enough since if $\phi\left(M_{0}\right)=1$ then by Lemma 2.4 we can write $M_{0} \sim 2 F_{1}+F_{2}$ with $F_{i}>0, F_{i}^{2}=0$ whence the contradiction $3 \phi(L) \leq M_{0} . L \leq k+2 \leq 2 \phi(L)+1$.

By Remark 4.9 with $b=k-1$ we see that we can apply Proposition 3.1

Let $C \in|L|$ be a smooth curve of gonality $k$ and let $\left|A_{C}\right|$ be a $g_{k}^{1}$ on $C$. Let $L \sim$ $N_{C}+N_{C}^{\prime}$ be the decomposition as in Proposition 3.1. Then $\mathcal{O}_{C}\left(N_{C}^{\prime}\right) \geq A_{C}$ and $\left|N_{C}^{\prime}\right|$ is base-component free, in particular $\left(N_{C}^{\prime}\right)^{2} \geq 0$.

We now show that we can assume that

$$
N_{C}^{2} \geq 0, L^{2} \geq 2 k+\left(N_{C}^{\prime}\right)^{2} \text { and } h^{0}\left(2 N_{C}^{\prime}-L+K_{S}\right) \leq 1 .
$$

Indeed, if we are in case (a) of Proposition 3.1, then either $N_{C} \cdot L \geq N_{C}^{\prime} . L$, whence $N_{C}^{2} \geq$ $\left(N_{C}^{\prime}\right)^{2} \geq 0$, or $\left|N_{C}\right|$ is base component-free, whence again $N_{C}^{2} \geq 0$. We also have that $\left(N_{C}^{\prime}\right)^{2} \leq k$ whence $L^{2} \geq 4 k-2 \geq 2 k+\left(N_{C}^{\prime}\right)^{2}$. Finally, $H$. $\left(N_{C}-N_{C}^{\prime}\right) \geq 0$ for an ample $H$, whence $h^{0}\left(2 N_{C}^{\prime}-L+K_{S}\right) \leq 1$. 
If we are in case (b) of Proposition 3.1, then $L^{2}=4 k-2$, so that by our assumptions there is a decomposition $L \sim M+N$ as in (ii). By Lemma 4.7(b) we can assume that $h^{1}\left(M+K_{S}\right)=h^{1}(N)=0$. Since

$$
\frac{1}{2}\left(M^{2}+\left(N+K_{S}\right)^{2}\right)+2=\frac{1}{2}\left(L^{2}-2 M . N\right)+2 \geq k+1,
$$

we have that either $h^{0}\left(\mathcal{E}\left(C, A_{C}\right)(-M)\right)>0$ or $h^{0}\left(\mathcal{E}\left(C, A_{C}\right)\left(-N+K_{S}\right)\right)>0$ by Lemma 3.2. It follows by Proposition 3.1(b) that we can assume that either $N_{C} \geq M$ or $N_{C} \geq N+K_{S}$. In the first case we get

$$
k-1 \leq M . N \leq M \cdot L \leq N_{C} \cdot L=N_{C}^{2}+N_{C} \cdot N_{C}^{\prime}=N_{C}^{2}+k
$$

showing that $N_{C}^{2} \geq 0$. Similarly we get $N_{C}^{2} \geq 0$ also when $N_{C} \geq N+K_{S}$. Moreover, we have that $N_{C} \cdot N_{C}^{\prime}=k$ whence $L^{2}=N_{C}^{2}+\left(N_{C}^{\prime}\right)^{2}+2 k \geq 2 k+\left(N_{C}^{\prime}\right)^{2}$. Finally, we note that $h^{0}\left(2 N_{C}^{\prime}-L+K_{S}\right)=h^{0}(R)=1$.

We have therefore proved that we can assume (28). In particular, by Lemma 4.7(b) we see that we are done if $h^{1}\left(N_{C}+K_{S}\right) \neq 0$. Therefore we can assume that $h^{1}\left(N_{C}+K_{S}\right)=0$.

Since there are only finitely many effective decompositions of $L$ we see that if $\mathcal{U}=$ $|L|_{s m}-\Sigma_{k-1}$, we can find a $\left(\mathcal{U}, L, k,\left\{A_{C}\right\}_{C \in \mathcal{U}}\right)$-dominant set of divisors $\left\{D_{1}, \ldots, D_{n}\right\}$ all of the form $D_{j}=N_{C}^{\prime}$ for some $C \in \mathcal{U}$. Also we have $h^{0}\left(2 D_{j}-L+K_{S}\right) \leq 1,\left(L-D_{j}\right)^{2} \geq 0$ and $L^{2} \geq 2 k+D_{j}^{2}$ for all $j$ by (28), whence we are done by Proposition 4.8.

Lemma 4.11. Let $S$ be an Enriques surface and let $|L|$ be a base-point free complete linear system on $S$. Suppose that a general smooth curve in $|L|$ has gonality $k \geq 3$ with $L^{2}=4 k-4$. Assume that there is an effective nontrivial decomposition $L \sim M+N$ such that $0 \leq M^{2} \leq 2 k-2,0 \leq N^{2} \leq 2 k-2, M . N \leq k-1, h^{0}\left(2 M-L+K_{S}\right) \leq 1$ and $h^{0}\left(2 N-L+\bar{K}_{S}\right) \leq 1$. Then $k \geq \min \{2 \phi(L), \mu(L)\}$.

Proof. As in the proof of the previous lemma we can assume $k \leq 2 \phi(L)-1$ and $h^{1}(M)=$ $h^{1}\left(M+K_{S}\right)=h^{1}(N)=h^{1}\left(N+K_{S}\right)=0$.

We will prove the existence of a line bundle $B>0$ such that $B^{2}=4$ and $B . L \leq k+2$.

Let $\mathcal{U}=|L|_{s m}-\Sigma_{k-1}$ and, for every $C \in \mathcal{U}$, let $\left|A_{C}\right|$ be a $g_{k}^{1}$ on $C$. Note that $\mathcal{U}$ has generic gonality $k$. As in the previous proof, by Lemma 3.2, we get that either $N_{\mid C} \geq A_{C}$ or $\left(M+K_{S}\right)_{\mid C} \geq A_{C}$.

Claim 4.12. Suppose that $N_{\mid C} \geq A_{C}$. Then $h^{0}(N) \geq 2$. Let $N_{0}$ be the moving part of $|N|$. Then $L-N_{0}>0, H^{1}\left(N_{0}-L\right)=0, N_{0}^{2}=N^{2}>0$ and $N_{0 \mid C} \geq A_{C}$.

Proof. We have $h^{0}(N)=h^{0}\left(N_{\mid C}\right) \geq h^{0}\left(A_{C}\right)=2$. Write $N \sim \Delta+N_{0}$ for some $\Delta \geq$ 0 . Obviously $L-N_{0}>0$. One relatively easily checks that $N_{0 \mid C} \geq A_{C}$, and using $h^{1}(N-L)=0$, that the map $H^{0}\left(N_{0}\right) \rightarrow H^{0}\left(N_{0 \mid C}\right)$ is surjective, whence an isomorphism. Now if $N_{0}^{2}>0$ then $h^{1}\left(N_{0}\right)=0$, whence $H^{1}\left(N_{0}-L\right)=0$ and $N_{0}^{2}=N^{2}>0$. If $N_{0}^{2}=0$, then $h^{0}\left(N_{0 \mid C}\right)=h^{0}\left(N_{0}\right)=2$ by [CD, proof of Cor.3.1.2], therefore $N_{0 \mid C} \sim A_{C}$. But then we get the contradiction $2 \phi(L) \leq N_{0} \cdot L=k$.

Conclusion of the proof of Lemma 4.11, Let $M_{0}$ be the moving part of $\left|M+K_{S}\right|$. Since a claim similar to Claim 4.12 holds if $\left(M+K_{S}\right)_{\mid C} \geq A_{C}$, we deduce that the pair $\left\{M_{0}, N_{0}\right\}$ is a $\left(\mathcal{U}, L, k,\left\{A_{C}\right\}_{C \in \mathcal{U}}\right)$-dominant set of divisors. Moreover $h^{0}\left(2 M_{0}-L+K_{S}\right) \leq h^{0}(2 M-$ $\left.L+K_{S}\right) \leq 1$ and $h^{0}\left(2 N_{0}-L+K_{S}\right) \leq h^{0}\left(2 N-L+K_{S}\right) \leq 1$ by assumption. To finish the proof, by Proposition 4.8, we need to show that $\left(L-N_{0}\right)^{2} \geq 0$ and $N_{0}^{2}+2 k-2 \leq L^{2}$ (and similarly for $M_{0}$ ).

By Claim 4.12 we get $N_{0}^{2}+2 k-2=N^{2}+2 k-2 \leq 4 k-4=L^{2}$ and $N_{0} \cdot\left(L-N_{0}\right)=$ $N_{0} \cdot L-N^{2} \leq N . L-N^{2}=N \cdot M \leq k-1$, from which $\left(L-N_{0}\right)^{2} \geq 0$ easily follows. 
We conclude this section by giving a few applications of the results obtained. They will be used to prove some cases of Theorem 1, to complete a theorem about plane curves in [KL2] and they will also be needed for the study of Gaussian maps in [KL3].

\subsection{The strategy in low genus.}

We investigate here the generic gonality of some line bundles of small genus. The strategy that we will employ is as follows.

Recall that $\Sigma_{s}=\left\{C \in|L|_{s m}: \operatorname{gon}(C) \leq s\right\}$. To prove that for a general curve $C_{\eta} \in|L|$ we have that gon $\left(C_{\eta}\right) \geq k+1$, we will assume that $\Sigma_{k}=|L|_{s m}$ and derive a contradiction. To this end we will consider the nonempty open subset $\mathcal{U}=|L|_{s m}-\Sigma_{k-1}$. Then for every $C \in \mathcal{U}$ we will have that $\operatorname{gon}(C)=k$ and, in all applications, $\mathcal{U}$ will have generic gonality $k$. We will find, in some cases with the help of of Proposition 3.1. a $\left(\mathcal{U}, L, k,\left\{A_{C}\right\}_{C \in \mathcal{U}}\right)$-dominant set of divisors that, together with Proposition 4.6, will lead to the desired contradiction.

Proposition 4.13. Let $|L|$ be a base-point free complete linear system on an Enriques surface $S$ with $L^{2}=10$. Then a general smooth curve $C \in|L|$ has $\operatorname{gon}(C)=4$ and is not isomorphic to a smooth plane quintic.

Remark 4.14. Note that curves isomorphic to a smooth plane quintic do occur (on a proper closed subset) by [St, $\mathrm{Um}$ if $\phi(L)=2$. On the other hand no smooth curve can be isomorphic to a smooth plane curve of degree at least 6 by the results in [KL2].

Proof. Since $|L|$ is base-point free we have $2 \leq \phi(L) \leq 3$. Moreover, if $B>0$ is a line bundle such that $B^{2}=4$ then $B . L \geq 7$ by the Hodge index theorem, whence $\mu(L) \geq 5$.

As is well-known, for any smooth $C \in|L|$ we have $\operatorname{gon}(C) \leq\left\lfloor\frac{9}{2}\right\rfloor=4$. We will now prove that a general such curve cannot be trigonal. Suppose in fact that this is the case. By Lemmas 2.17 and 2.18 we see that we can apply Lemma4.10 with $k=3, M=E, N=L-E$, and we get the contradiction $3 \geq \min \{2 \phi(L), \mu(L)\} \geq 4$.

Now we show that $C$ is not isomorphic to a smooth plane quintic.

Suppose first that $\phi(L)=2$. From Lemma 2.17 and Theorem 2.6, we easily see that $\left|\mathcal{O}_{C}\left(E+E_{1}\right)\right|$ is a complete $g_{5}^{1}$ on $C$.

If $C$ is isomorphic to a smooth plane quintic then it has no base-point free complete $g_{5}^{1}$. Hence $\left|\mathcal{O}_{C}\left(E+E_{1}\right)\right|$ must have base points and it follows that $C$ passes through one of the two base points of $\left|E+E_{1}\right|$. But this cannot happen for general $C \in|L|$.

Now suppose that $\phi(L)=3$ and use Lemma 2.18 .

By what we have just proved the open subset $\mathcal{U}^{\prime}:=|L|_{s m}-\Sigma_{3}$ is nonempty. Consider now, in $|L|_{s m}$, the closed subset $\Sigma_{5}^{2}=\left\{C \in|L|_{s m}: C\right.$ has a $\left.g_{5}^{2}\right\}$. We will prove that $\Sigma_{5}^{2} \cap \mathcal{U}^{\prime} \subset \mathcal{U}^{\prime}$ is a strict inclusion. This will give that on the nonempty open subset $\mathcal{U}^{\prime}-\Sigma_{5}^{2} \cap \mathcal{U}^{\prime}$ every curve is not trigonal and is not isomorphic to a plane quintic.

Suppose that $\Sigma_{5}^{2} \cap \mathcal{U}^{\prime}=\mathcal{U}^{\prime}$. Then every $C \in \mathcal{U}^{\prime}$ has a $g_{5}^{2}$. Since $C$ has genus 6 , we get that the $g_{5}^{2}$ must be very ample, therefore every $C \in \mathcal{U}^{\prime}$ is isomorphic to a plane quintic.

Now certainly $\mathcal{U}^{\prime}$ has generic gonality 4 . We now construct a particular $g_{4}^{1}$ on each $C \in \mathcal{U}^{\prime}$.

By Lemma 2.18(i), $\left|\mathcal{O}_{C}\left(E_{1}+E_{2}\right)\right|$ is a complete base-point free $g_{7}^{2}$. As $C$ cannot be isomorphic to a smooth plane septic for reasons of genus, it follows that $\mathcal{O}_{C}\left(E_{1}+E_{2}\right)$ is not very ample. Since in addition any complete $g_{5}^{1}$ on $C$ must have a base point, there exists an effective divisor $Z_{3}$ of degree 3 on $C$ such that $\left|A_{C}:=\mathcal{O}_{C}\left(E_{1}+E_{2}\right)\left(-Z_{3}\right)\right|$ is a complete $g_{4}^{1}$ on $C$, which has to be base-point free, since gon $(C)=4$.

By Lemma2.18(i), it is easily seen that $D$ is $\left(\mathcal{U}^{\prime}, L, 4,\left\{A_{C}\right\}_{C \in \mathcal{U}^{\prime}}\right)$-dominant. Now $D^{2}=4$, $D . L=7$ and $h^{1}(L-2 D) \leq 1$ by Lemma 2.18(ii). By Proposition 4.6 we see that equality occurs in (18). But now Proposition 4.6(c) yields a contradiction. 
Proposition 4.15. Let $|L|$ be a base-point free complete linear system on an Enriques surface $S$ with $L^{2} \geq 14$ and $\phi(L)=2$. Then a general smooth curve $C \in|L|$ has gonality 4 and possesses a unique $g_{4}^{1}$, which is cut out by a unique genus one pencil on $S$. Moreover $W_{4}^{1}(C)$ is smooth when $L^{2}=14$.

Remark 4.16. In fact $W_{4}^{1}(C)$ is smooth also when $L^{2}=16$. This will be proved in [KL2].

Proof. It is easy to see that there is a unique genus one pencil $|2 E|$ such that $E . L=\phi(L)$, thus also cutting out a $g_{4}^{1}$ on $C$.

Now by GLM, Thm.1.4] we know that $\operatorname{gon}(C)>3$, whence gon $(C)=4=2 \phi(L)$. Moreover, again by [GLM, Thm.1.4(1.7)], we know that, if $L^{2} \geq 18$, then every $g_{4}^{1}$ is cut out by a genus one pencil on $S$ and we are done in this case.

Let us now treat the case $L^{2}=14$.

Consider the nonempty open subset $\mathcal{U}=|L|_{s m}-\Sigma_{3}$ and suppose, to get a contradiction, that $\mathcal{U}$ has generic gonality 4 , that is that every $C \in \mathcal{U}$ has a $g_{4}^{1}=\left|A_{C}\right|$ which is not cut out by a genus one pencil on $S$.

Letting $L \sim 3 E+E_{1}+E_{2}$ be as in Lemma 2.19, we have that $|D|:=\left|3 E+E_{1}\right|$ is base-component free by [CD, Prop.3.1.6]. By Riemann-Roch $h^{1}\left(E_{2}+K_{S}\right)=0$, whence, for every $C \in \mathcal{U}$, either $D_{\mid C} \geq A_{C}$ or $\left(E_{2}+K_{S}\right)_{\mid C} \geq A_{C}$ by Lemma 3.2. In the second case we get a contradiction since $h^{0}\left(\left(E_{2}+K_{S}\right)_{\mid C}\right)=h^{0}\left(E_{2}+K_{S}\right)=1$. As $|D|$ is base-component free, we have that $D$ is $\left(\mathcal{U}, L, k,\left\{A_{C}\right\}_{C \in \mathcal{U}}\right)$-dominant. But $\phi(D)=E . D=1$, contradicting Proposition 4.6(a).

Therefore there is a curve $C_{0} \in|L|_{s m}$ such that every $g_{4}^{1}$ on $C_{0}$ is cut out by a genus one pencil on $S$, which is unique by what we said above.

Let $A=(2 E)_{\mid C_{0}}$ be the unique $g_{4}^{1}$ on $C_{0}$. We will prove that $\mu_{0, A}$ is surjective. As is well-known [ACGH, Prop.IV.4.2] this means that $W_{4}^{1}\left(C_{0}\right)$ is smooth at its unique point $A$ and therefore the same holds in an open neighborhood of $C_{0}$ in $|L|$.

To see the surjectivity of $\mu_{0, A}$ we observe that, as $C_{0}$ is nontrigonal, if $\mu_{0, A}$ is not surjective then, by the base-point-free pencil trick we have that $h^{0}\left(\omega_{C_{0}}-2 A\right) \geq 3$. Since $\operatorname{deg}\left(\omega_{C_{0}}-2 A\right)=6$ and $\operatorname{Cliff}\left(C_{0}\right)=2$ we deduce that $\left|\omega_{C_{0}}-2 A\right|$ is a base-point free $g_{6}^{2}$ on $C_{0}$. Let $\varphi: C_{0} \rightarrow X \subset \mathbb{P}^{2}$ be the morphism defined by $\left|\omega_{C_{0}}-2 A\right|$. If $\varphi$ is not birational, since $C_{0}$ is nontrigonal, then $\varphi$ is a cover of degree 2 of a smooth plane cubic $X$, whence $C_{0}$ is bielliptic. But this is excluded since $C_{0}$ has a unique $g_{4}^{1}$. Therefore $\varphi$ is birational, and again, since $C_{0}$ is nontrigonal, the image $X$ is a plane sextic with two (possibly infinitely near) double points. Hence $C_{0}$, in its canonical embedding, is isomorphic to a quadric section of a Del Pezzo surface, namely the anticanonical embedding of the plane blown-up at two points. But in [KL3, Lemma5.13] it is proved that this case cannot occur.

Next we treat the case $L^{2}=16$.

Consider the nonempty open subset $\mathcal{U}=|L|_{s m}-\Sigma_{3}$ and the finitely many base-component free line bundles $L^{\prime}$ such that $0 \leq L^{\prime} \leq L$ and Bs $\left|L^{\prime}\right| \neq \emptyset$. We shrink $\mathcal{U}$, if necessary, to a nonempty open subset $\mathcal{U}^{\prime} \subseteq \mathcal{U}$ by removing the finitely many closed subsets given by curves $C \in \mathcal{U}$ such that $C \cap \mathrm{Bs}\left|L^{\prime}\right| \neq \emptyset$ for some $L^{\prime}$ as above.

Let $C \in \mathcal{U}^{\prime}$, let $A_{C}$ be a $g_{4}^{1}$ on $C$ and apply Proposition 3.1 to $A_{C}$. Then we must be in case (a) of the same proposition and $L \sim N_{C}+N_{C}^{\prime}$ with the three possibilities $\left(N_{C}^{\prime}\right)^{2}=0$ with $N_{C}^{\prime} \cdot L \leq 4$, or $\left(N_{C}^{\prime}\right)^{2}=2$ with $N_{C}^{\prime} \cdot L \leq 6$, or $\left(N_{C}^{\prime}\right)^{2}=4$ with $N_{C}^{\prime} \cdot L=8$.

In the first case $\left|N_{C}^{\prime}\right|$ is a genus one pencil such that $A_{C} \sim \mathcal{O}_{C}\left(N_{C}^{\prime}\right)$. In the second case the Hodge index theorem yields $N_{C}^{\prime} . L=6$. But $\left|N_{C}^{\prime}\right|$ has two base points and by Proposition 3.1 we have $\mathrm{Bs}\left|N_{C}^{\prime}\right| \cap C \neq \emptyset$. In the third case the Hodge index theorem yields 
$L \equiv 2 N_{C}^{\prime}$ whence $\phi\left(N_{C}^{\prime}\right)=1$. But now Proposition 3.1 implies that $C \cap \operatorname{Bs}\left|N_{C}^{\prime}\right| \neq \emptyset$, a contradiction.

Hence on any curve $C \in \mathcal{U}^{\prime}$ there is a unique $g_{4}^{1}$, namely $A=(2 E)_{\mid C}$.

\section{The Proof of Theorem 1}

Lemma 5.1. Let $|L|$ be a base-component free complete linear system on an Enriques surface $S$ with $L^{2}>0$ and let $C \in|L|_{\text {sm }}$. Then $\operatorname{gon}(C) \leq \min \left\{2 \phi(L), \mu(L),\left\lfloor\frac{L^{2}}{4}\right\rfloor+2\right\}$.

Proof. We can of course assume $L^{2} \geq 4$ and that $k:=\mu(L) \leq 2 \phi(L)-1$ and $L^{2} \geq 4 k-4$. By Proposition 2.8 and Lemma 2.14 we have $k \geq 10$ and $L^{2} \geq 36$. Pick $B>0$ with $B^{2}=4$ and $B . L=k+2$. Then Riemann-Roch yields $h^{0}(L-B) \geq 9$. By Lemma 4.7(b) we have $h^{1}(B)=h^{1}\left(L-B+K_{S}\right)=0$, for otherwise we can find a line bundle $B_{0}>0$ such that $B_{0}^{2}=4$ and $B_{0} . L \leq k$. Now if $\phi\left(B_{0}\right)=1$ then, by Lemma 2.4 we can write $B_{0} \sim 2 F_{1}+F_{2}$ with $F_{i}>0, F_{i}^{2}=0, i=1,2$, whence $3 \phi(L) \leq B_{0} . L \leq k \leq 2 \phi(L)-1$, a contradiction. Therefore $\phi\left(B_{0}\right)=2$, but $B_{0} . L-2<k$, a contradiction.

Hence $h^{0}(B)=h^{0}\left(\mathcal{O}_{C}(B)\right)=3$. Now $\mathcal{O}_{C}(B)$ cannot be very ample, for reasons of genus and from the Hodge index theorem applied to $B$ and $L$. Therefore gon $(C) \leq k=\mu(L)$.

Proof of Theorem 1. By Lemma 5.1 we need to show that

$$
\operatorname{gon}(C) \geq \min \left\{2 \phi(L), \mu(L),\left\lfloor\frac{L^{2}}{4}\right\rfloor+2\right\}
$$

holds for a general curve $C \in|L|$.

Now (29) holds if $\phi(L)=1$. Therefore we can assume $\phi(L) \geq 2$ and $L^{2} \geq 4$. If $4 \leq L^{2} \leq 6$ the Hodge index theorem shows that $\mu(L) \geq 3$, whence $\min \left\{2 \phi(L), \mu(L),\left\lfloor\frac{L^{2}}{4}\right\rfloor+2\right\}=3$ and a general curve in $|L|$ is trigonal by [CD, Prop.3.1.4, Prop.4.5.1, Thm.4.5.4 and Rmk.4.5.2].

We can therefore assume that $L^{2} \geq 8$ and that if $k$ is the gonality of a general curve in $|L|$ then $k \geq 3$. Moreover we can also assume that $L^{2} \geq 4 k-4$ and $k \leq 2 \phi(L)-1$. By Lemma 4.10 we are left with the cases

$$
L^{2}=4 k-2 \text { or } 4 k-4, \text { and } k \leq 2 \phi(L)-1 .
$$

Hence $4 k-2 \geq L^{2} \geq(\phi(L))^{2} \geq \frac{(k+1)^{2}}{4}$ gives $k \leq 13$ and $L^{2} \leq 50$. By Proposition 1 we deduce that $k \leq 11$ and $L^{2} \leq 42$.

We will prove (29) by finding, for each case in (30), an effective decomposition $L \sim$ $M+N$ satisfying the conditions in Lemma 4.10 or Lemma 4.11 and then applying those lemmas. In each case we will first use (30) to determine $\phi(L)$ and then Lemma 2.4 to find a decomposition of $L$. Then we state what $M$ is, leaving most verifications to the reader.

5.1. Notation. In the sequel of the proof we will let $E>0$ be such that $E^{2}=0$ and $E . L=\phi(L)$. Moreover, any $E^{\prime}, E_{1}, E_{2}$, etc., will be effective, nonzero isotropic divisors.

5.2. $k \leq 4$ and $L^{2}=4 k-2$. The possibilities are $\left(L^{2}, \phi(L), k\right)=(10,2,3),(10,3,3)$ or $(14,3,4)$, and we are done by Lemma 4.10 setting $M=E$.

5.3. $k=3$ and $L^{2}=8$. We have $\phi(L)=2$ and $L \sim 2 E+E^{\prime}$ with $E^{\prime} . E=2$, and one easily sees that either $\phi(L-E)=2$ and $E^{\prime}$ is primitive or $\phi(L-E)=1$ and $E^{\prime} \equiv 2 E_{1}$. In the first case set $M=E$ and in the second $M=E+E_{1}$. 
5.4. $k=4$ and $L^{2}=12$. We have $\phi(L)=3$. Choose a maximal $E$ such that $E . L=3$. We have $L \sim 2 E+E_{1}$ with $E . E_{1}=3$ and one easily sees that $E_{1}$ is primitive. We set $M=E$ and show how to verify that $h^{0}\left(2 N-L+K_{S}\right)=h^{0}\left(E_{1}+K_{S}\right)=1$. By Riemann-Roch and Theorem 2.6] it suffices to show that $E_{1}$ is quasi-nef.

Assume, to get a contradiction, that there is a $\Delta>0$ such that $\Delta^{2}=-2$ and $\Delta \cdot E_{1} \leq-2$. Set $m=\Delta \cdot E_{1} \geq 2$. By Lemma 2.3 there is a primitive $A>0$ such that $A^{2}=0$ and $E_{1} \sim A+m \Delta$. We have $0 \leq L . \Delta=2 E . \Delta-m$ by the nefness of $L$, whence $2 E . \Delta \geq m$. From $3=E \cdot E_{1}=E \cdot A+m E . \Delta$ we get that $m=2, E \cdot \Delta=E \cdot A=1$. Therefore we can write $L \sim 2(E+\Delta)+A$, contradicting the maximality of $E$.

5.5. $k=5$ and $L^{2}=16$. We have $\phi(L)=3$ or 4 . If $\phi(L)=3$, let $L \sim 2 E+E_{1}+E_{2}$ be as in Lemma 2.20. If $h^{0}\left(2 E+E_{1}-E_{2}\right)=h^{0}\left(2 E+E_{1}-E_{2}+K_{S}\right)=1$ we set $M=E_{2}$. Otherwise, by Lemma 2.20, we must have that $E_{1} \equiv 2 F$ and we set $M=E+F+E_{2}$. (Note that $h^{0}\left(2 M-L+K_{S}\right)=1$ since $\left(2 M-L+K_{S}\right) \cdot L=E_{2} . L<2 \phi(L)$.)

If $\phi(L)=4$, by Lemma 2.11 we have $L \equiv 2\left(E+E^{\prime}\right)$, with $E \cdot E^{\prime}=2$ and we set $M=E+E^{\prime}$.

5.6. $k=5$ and $L^{2}=18$. We have $\phi(L)=3$ or 4 . If $\phi(L)=4$, set $M=E$. If $\phi(L)=3$ and $\phi(L-2 E)=1$, then $L \sim 3\left(E+E_{1}\right)$ with $E . E_{1}=1$ and we set $M=2 E+E_{1}$. If $\phi(L)=3$ and $\phi(L-2 E)=2$, then $L-2 E \sim E_{1}+E_{2}+E_{3}$, with $E_{i} . E_{j}=E . E_{i}=1$, for all $i \neq j$, and we set $M=E+E_{1}+E_{2}$.

5.7. $k=6$ and $L^{2}=20$. We have $\phi(L)=4$ and it is easily seen that we can write $L \sim E_{0}+E_{1}+E_{2}+E_{3}+E_{4}$, with $E_{0}:=E$ and $E_{i} . E_{j}=1$ for $i \neq j$. Therefore $\left\{E_{0}, \ldots, E_{4}\right\}$ is an isotropic 5-sequence and by [CD, Cor.2.5.6] we can find a divisor $F$ such that $F^{2}=0$, $F . E_{i}=1$ for $0 \leq i \leq 4$. Then $F . L=5$. Among all such $F$ 's we choose a maximal one and let this be $M$. (We show how to see that $h^{0}\left(2 N-L+K_{S}\right)=h^{0}\left(L+K_{S}-2 M\right)=1$ : Note that since $\left(L+K_{S}-2 M\right)^{2}=0$ and $\left(L+K_{S}-2 M\right) \cdot L=10$, we have that $L+K_{S}-2 M>0$ and it is easily seen to be primitive. Therefore, by Theorem 2.6, it suffices to show that $L+K_{S}-2 M$ is quasi-nef. Assume there is a $\Delta>0$ such that $\Delta^{2}=-2$ and $\Delta \cdot\left(L+K_{S}-2 M\right) \leq-2$. Then $\Delta . M \geq 1$ by the nefness of $L$ and if $\Delta . M=1$ we get $\Delta . L=0$ whence $(M+\Delta)^{2}=0$, $(M+\Delta) \cdot L=5$, contradicting the maximality of $M$. Therefore $\Delta \cdot M \geq 2$ and $(M+\Delta)^{2} \geq 2$ whence $h^{0}(M+\Delta) \geq 2$ and we get $5+\Delta \cdot L=(M+\Delta) \cdot L \geq 2 \phi(L)=8$, thus giving $\Delta . L \geq 3$. Now the nefness of $L$ implies that $\Delta . M \geq 3$, so that $(2 M+\Delta)^{2} \geq 10$. Also $(2 M+\Delta) \cdot(L-2 M-\Delta)=12+\Delta \cdot(L-2 M)-2 \Delta \cdot M \leq 4$ and $(2 M+\Delta) \cdot(L-2 M-\Delta) \geq 0$ by Lemma 2.2 since $L-2 M-\Delta>0$ by Lemma 2.3. Now the Hodge index theorem on $2 M+\Delta$ and $L-2 M-\Delta$ yields a contradiction.

5.8. $k=6$ and $L^{2}=22$. We have $\phi(L)=4$ and it is easily seen that $L \sim 2 E+E_{1}+E_{2}+E_{3}$ with $E . E_{1}=2, E . E_{i}=1$ for $i=2,3, E_{i} . E_{j}=1$ for $1 \leq i<j \leq 3$. Then we set $M=E_{1}$.

5.9. $k=7$ and $L^{2}=24$. We have $\phi(L)=4$. One easily verifies that $(L-2 E)^{2}=8$ and $\phi(L-2 E)=2$, so that $L-2 E \sim 2 E_{1}+E_{2}$ with $E_{1}$ primitive and $E_{1} . E_{2}=2$.

We have $4=2 E . E_{1}+E . E_{2}$. Hence, by Lemma 2.2, we must have $E . E_{1}=1$ or 2 . In the latter case we get $E . E_{2}=0$, then $E \equiv q E_{2}$ for some $q \geq 1$ by Lemma 2.2. From $E_{1} \cdot E=E_{1} \cdot E_{2}=2$ we get that $E \equiv E_{2}$ and we can set $M=2 E+E_{1}$.

If $E \cdot E_{1}=1$ and $E \cdot E_{2}=2$, setting $B=E+E_{1}$ and $A=E_{2}$, we have a decomposition

$$
L \sim 2 B+A \text {, with } A^{2}=0, B^{2}=2 \text { and } A \cdot B=4 .
$$

Now among all effective decompositions of the form (31) pick one such that $B$ is maximal. As in case 5.4 one easily verifies that $A$ is quasi-nef. If $h^{1}\left(A+K_{S}\right)>0$, by Theorem 2.6 and 
the fact that $A . L=2 \phi(L)$, we have that $A \equiv 2 A^{\prime}$ for some divisor $A^{\prime}>0$ with $\left(A^{\prime}\right)^{2}=0$. In this case $L \equiv 2 M$, setting $M=B+A^{\prime}$. If $h^{1}\left(A+K_{S}\right)=0$ we set $M=B+A$.

5.10. $k=7$ and $L^{2}=26$. By (30) and Proposition 1 1 we get $\phi(L)=4$. We have $L-3 E \sim$ $E_{1}+E_{2}$, with $E_{1} \cdot E_{2}=1$. By symmetry we have the two possibilities $\left(E \cdot E_{1}, E \cdot E_{2}\right)=(2,2)$ and $(1,3)$. We set $M=2 E+E_{2}$.

5.11. $k=8$ and $L^{2}=28$. We have $\phi(L)=5$. By Proposition 1 and Lemma 2.14 we can write $L \sim 2 E+2 E_{1}+E_{2}$ with $E . E_{1}=E_{1} \cdot E_{2}=2$ and $E . E_{2}=1$. We set $M=E+E_{1}+E_{2}$. (Note that $h^{0}\left(2 M-L+K_{S}\right)=h^{0}\left(E_{2}+K_{S}\right)=1$, since $E_{2} \cdot L=6<2 \phi(L)$.)

5.12. $k=8$ and $L^{2}=30$. We have $\phi(L)=5$ and $L \sim 2 E+E_{1}+E_{2}+E_{3}$, with $E_{1} . E_{2}=1$, $E_{1} \cdot E_{3}=E_{2} \cdot E_{3}=2$ and the three possibilities $\left(E \cdot E_{1}, E \cdot E_{2}, E \cdot E_{3}\right)=(1,1,3),(1,2,2)$ and $(2,2,1)$. In the first case we set $M=2 E+E_{3}$ and in the two latter $M=E_{2}$.

5.13. $k=9$ and $L^{2}=32$. We have $\phi(L)=5$ and $L \sim 3 E+E_{1}+E_{2}$, with $E . E_{1}=2$, $E . E_{2}=3$ and $E_{1} \cdot E_{2}=1$. We set $M=2 E+E_{2}$. (Note that $h^{0}\left(2 M-L+K_{S}\right) \leq 1$ as $\left.\left(2 M-L+K_{S}\right) \cdot L=8<2 \phi(L).\right)$

5.14. $k=9$ and $L^{2}=34$. We have $\phi(L)=5$ and one easily shows that $\phi(L-2 E)=3$, so that $L-2 E \sim 2 E_{1}+E_{2}+E_{3}$, with $E_{1} \cdot E_{3}=2$ and $E_{1} \cdot E_{2}=E_{2} \cdot E_{3}=1$. Also either $E . E_{1}=2$ and $L \equiv 3 E+2 E_{1}+E_{2}$, in which case we set $M=2 E+E_{1}$ or $E . E_{1}=1$, $\left(E . E_{2}, E . E_{3}\right)=(1,2)$ or $(2,1)$ and we set $M=E+E_{1}+E_{2}$.

5.15. $k=10$ and $L^{2}=36$. We have $\phi(L)=6$. By Lemmas 2.11 and 2.14 we get $k=$ $\mu(L)=10$ and we are done.

5.16. $k=10$ and $L^{2}=38$. This case cannot exist by (30) and Proposition 1,

5.17. $k=11$ and $L^{2}=40$. We have $\phi(L)=6$. By Proposition 1 and Lemma 2.14 we have that either $L \sim 3 E+2 E_{1}+E_{2}$ with $E \cdot E_{1}=E \cdot E_{2}=2, E_{1} \cdot E_{2}=1$ or $L \equiv 2 D$ for a $D>0$ with $D^{2}=10$. In the second case we set $M=D$. In the first case we set $M=2 E+2 E_{1}$. (Note that $h^{0}\left(2 M-L+K_{S}\right)=h^{0}\left(E+2 E_{1}-E_{2}+K_{S}\right) \leq 1$ since $\left.\left(E+2 E_{1}-E_{2}+K_{S}\right) \cdot\left(E+E_{1}\right)=3<2 \phi\left(E+E_{1}\right).\right)$

5.18. $k=11$ and $L^{2}=42$. We have $\phi(L)=6$ and one can check that $\phi(L-2 E)=4$. Then $L-2 E \sim 2 E_{1}+E_{2}+E_{3}$, with $E_{1} \cdot E_{2}=E_{1} \cdot E_{3}=2$ and $E_{2} \cdot E_{3}=1$.

From $6=E . L=2 E . E_{1}+E . E_{2}+E . E_{3}$ we find $E . E_{2} \leq 3$ and $E . E_{3} \leq 3$, but if $E . E_{i}=3$ for $i=2$ or 3 , then $\left(E+E_{i}\right)^{2}=6$ yields the contradiction $3 \phi(L)=18 \leq\left(E+E_{i}\right) \cdot L=17$. Hence $E . E_{2} \leq 2$ and $E . E_{3} \leq 2$, so that $\left(E . E_{1}, E . E_{2}, E . E_{3}\right)=(1,2,2)$ or $(2,1,1)$. We set $M=2 E+E_{1}+E_{2}$.

This concludes the proof of (29), whence that of Theorem 1.

Proof of Corollary 1. Apply Theorem 1, Propositions 1 and 2.8, Lemmas 2.14 and 2.16. 


\section{The minimal gonality of A SMOoth CURVE IN A COMPLETE LiNEAR System}

In this section we prove Corollary 2 and give some examples.

Proof of Corollary 2. Let $k:=$ gengon $|L|$. Assume that there is a smooth curve $C_{0} \in|L|$ with $k_{0}:=\operatorname{gon}\left(C_{0}\right) \leq k-2$. Then $k_{0} \leq\left\lfloor\frac{L^{2}}{4}\right\rfloor$ by Lemma 5.1, so that $L^{2} \geq 4 k_{0} \geq 8$. By Remark 4.9 with $b=k_{0}-1$ we get $L^{2} \geq 2 k_{0}-2+2 \phi(L)$. Fix an ample divisor $H$ and let $A_{0}$ be a $g_{k_{0}}^{1}$ on $C_{0}$. By Proposition 3.1)(a) we obtain an effective nontrivial decomposition $L \sim N+N^{\prime}$ with $N . N^{\prime} \leq k_{0}$. Moreover we have $\left(N-N^{\prime}\right)^{2}=L^{2}-4 N . N^{\prime} \geq 0$, and $H$. $\left(N-N^{\prime}\right) \geq 0$, so that by Riemann-Roch we have that either $N \equiv N^{\prime}$ and $L \equiv 2 N^{\prime}$ or $L-2 N^{\prime}=N-N^{\prime} \geq 0$. Also recall that $h^{0}\left(N^{\prime}\right) \geq 2$. Consider the set

$X_{L}=\left\{D: h^{0}(D) \geq 2, D \cdot(L-D) \leq k_{0},(L-2 D)^{2} \geq 0\right.$ and either $L-2 D \geq 0$ or $\left.L \equiv 2 D\right\}$.

Now $X_{L} \neq \emptyset$ since $N^{\prime} \in X_{L}$, whence we can choose an element $D_{0} \in X_{L}$ for which $H . D_{0}$ is minimal. Note that $h^{0}\left(D_{0}\right) \geq 2$ implies that $D_{0} . L \geq 2 \phi(L)$.

If $D_{0}^{2} \geq 6$, then pick any $F>0$ such that $F^{2}=0$ and $F . D_{0}=\phi\left(D_{0}\right)$. Then $D_{0}-F$ is easily seen to contradict the minimality of $D_{0}$. Hence $D_{0}^{2} \leq 4$.

If $D_{0}^{2} \leq 2$ we get

$$
k_{0} \leq k-2 \leq 2 \phi(L)-2 \leq D_{0} \cdot L-2=D_{0}^{2}+D_{0} \cdot\left(L-D_{0}\right)-2 \leq k_{0}
$$

whence $k_{0}=k-2, D_{0}^{2}=2$ and $D_{0} \cdot L=2 \phi(L)$. Now the Hodge index theorem applied to $D_{0}$ and $L$ implies that $\phi(L) \geq\left\lceil\sqrt{\frac{L^{2}}{2}}\right\rceil$.

If $D_{0}^{2}=4$ we first show that if $\phi\left(D_{0}\right)=1$ then $L^{2}=8$ and $\phi(L)=2$, whence $\phi(L)=$ $\left\lceil\sqrt{\frac{L^{2}}{2}}\right\rceil$. If $\phi\left(D_{0}\right)=1$ we can write $D_{0} \sim 2 F_{1}+F_{2}$ with $F_{i}>0, F_{i}^{2}=0, i=1,2, F_{1} \cdot F_{2}=1$ so that

$$
3 \phi(L) \leq D_{0} \cdot L=D_{0}^{2}+D_{0} \cdot\left(L-D_{0}\right) \leq k_{0}+4 \leq k+2 \leq 2 \phi(L)+2
$$

whence $\phi(L) \leq 2$ and therefore $2 \leq k_{0} \leq k-2 \leq 2 \phi(L)-2 \leq 2$ giving $k=4, k_{0}=2$ and $\phi(L)=2$. Applying Proposition 3.1 to the hyperelliptic curve $C_{0}$ we get that $L \sim N_{0}+N_{0}^{\prime}$ with $\left(N_{0}^{\prime}\right)^{2} \leq 2$ and $L . N_{0}^{\prime} \leq 4$. If $\left(N_{0}^{\prime}\right)^{2}=0$ by [GLM, Lemma2.1] we have that $N_{0}^{\prime} \sim 2 E$ is a genus one pencil such that $(2 E)_{\mid C_{0}} \sim A_{0}$, that is $E . L=1$, a contradiction. Therefore $\left(N_{0}^{\prime}\right)^{2}=2$ and the Hodge index theorem implies that $L^{2} \leq 8$.

Now if $D_{0}^{2}=4$ and $\phi\left(D_{0}\right)=2$, we have, by Lemma 5.1 ,

$$
k \leq \mu(L) \leq D_{0} \cdot L-2=2+D_{0} \cdot\left(L-D_{0}\right) \leq 2+k_{0} \leq k
$$

whence $k_{0}=k-2$ and $\mu(L)=D_{0} . L-2=k \leq 2 \phi(L)$. The Hodge index theorem applied to $D_{0}$ and $L$ then gives $4 L^{2} \leq(2 \phi(L)+2)^{2}$ and it is esily checked that $\phi(L) \geq\left\lceil\sqrt{\frac{L^{2}}{2}}\right\rceil$.

6.1. A few examples. The flexibility of the Picard group of an Enriques surface allows us to give several examples to show the behavior of the gonality. We give two here, showing that all cases in Corollary 2 , mingon $|L|=$ gengon $|L|-2$, gengon $|L|-1$, gengon $|L|$ do occur.

Example 1. Let $\left|2 E_{i}\right|, i=1,2$ be two genus one pencils on an Enriques surface $S$ such that $E_{1} \cdot E_{2}=1$ and consider the line bundle

$$
L \sim a E_{1}+b E_{2} \text { for } b \geq a \geq 3 .
$$

It is easily checked that $2 \phi(L)=2 a \leq \min \left\{\mu(L),\left\lfloor\frac{L^{2}}{4}\right\rfloor+2\right\}$, so that, by Theorem 11, we have that $k:=$ gengon $|L|=2 a$. Moreover $2 \phi(L)<\mu(L)$. 
We recall that by [CD, Prop.3.1.6, 3.1.4 and Thm.4.4.1] the base locus of $\left|E_{1}+E_{2}\right|$ consists of two distinct points $x$ and $y$. Let $B \in\left|E_{1}+E_{2}\right|$ be general and consider the exact sequence

$$
0 \longrightarrow L-E_{1}-E_{2} \longrightarrow \mathcal{J}_{\{x, y\} / S} \otimes L \longrightarrow\left(L-E_{1}-E_{2}\right)_{\mid B} \longrightarrow 0,
$$

the base point freeness of $L-E_{1}-E_{2} \sim(a-1) E_{1}+(b-1) E_{2}$ (by [CD, Prop.3.1.6, 3.1.4 and Thm.4.4.1]) and the fact that $H^{1}\left(L-E_{1}-E_{2}\right)=0$, we get that the base-scheme of $\left|\mathcal{J}_{\{x, y\} / S} \otimes L\right|$ is $\{x, y\}$. Therefore, by Bertini, the general curves $C_{0} \in\left|\mathcal{J}_{\{x, y\} / S} \otimes L\right|$ and $C_{0}^{\prime} \in\left|\mathcal{J}_{\{x\} / S} \otimes L\right|$ are smooth and irreducible.

Suppose that $b=a$. In this case we claim that both $k_{0}=k-2$ and $k_{0}=k-1$ do occur as gonality of some smooth curve in $|L|$. Now $\left|\left(E_{1}+E_{2}\right)_{\mid C_{0}}(-x-y)\right|$ is a $g_{k-2}^{1}$ on $C_{0}$, while $\left|\left(E_{1}+E_{2}\right)_{\mid C_{0}^{\prime}}(-x)\right|$ is a $g_{k-1}^{1}$ on $C_{0}^{\prime}$. Therefore $k_{0}:=\operatorname{gon}\left(C_{0}\right)=k-2$ by Corollary 2 ,

To see that $k_{0}^{\prime}:=\operatorname{gon}\left(C_{0}^{\prime}\right)=k-1$ we we first note that $y \notin C_{0}^{\prime}$ and also that Bs $\mid E_{1}+E_{2}+$ $K_{S} \mid \cap C_{0}^{\prime}=\emptyset$. By Corollary 2 we can suppose that $k_{0}^{\prime}=k-2$ and let $A$ be a $g_{k-2}^{1}$ on $C_{0}^{\prime}$. Applying Proposition 3.1(a) we obtain an effective nontrivial decomposition $L \sim N+N^{\prime}$ with $\left|N^{\prime}\right|$ base-component free, $N^{\prime} . L \leq\left(N^{\prime}\right)^{2}+2 a-2 \leq 4 a-4$ and $N_{\mid C_{0}^{\prime}}^{\prime} \geq A$.

If $\left(N^{\prime}\right)^{2} \geq 6$ we get $3 a=3 \phi(L) \leq N^{\prime} . L \leq\left(N^{\prime}\right)^{2}+2 a-2$, so that $\left(N^{\prime}\right)^{2} \geq a+2$. But then the Hodge index theorem applied to $N^{\prime}$ and $L$ gives a contradiction.

If $\left(N^{\prime}\right)^{2}=4$ we get that $L . N^{\prime} \leq 2 a+2$, whence the contradiction $\mu(L) \leq 2 a=2 \phi(L)$. If $\left(N^{\prime}\right)^{2}=0,2$ we have that $2 a=2 \phi(L) \leq L . N^{\prime} \leq\left(N^{\prime}\right)^{2}+2 a-2$, whence $\left(N^{\prime}\right)^{2}=2$ and $N^{\prime} . L=2 a$, so that the Hodge index theorem implies that $L \equiv a N^{\prime}$, whence $N^{\prime} \equiv E_{1}+E_{2}$. On the other hand $h^{0}\left(N_{\mid C_{0}^{\prime}}^{\prime}\right)=h^{0}\left(N^{\prime}\right)=2$, since $h^{i}\left(N^{\prime}-L\right)=0$ for $i=0,1$. Therefore there are two points $x^{\prime}, y^{\prime} \in C_{0}^{\prime}$ such that $N_{\mid C_{0}^{\prime}}^{\prime} \sim A+x^{\prime}+y^{\prime}$ and we deduce that $x^{\prime}, y^{\prime} \in$ $\operatorname{Bs}\left|N_{\mid C_{0}^{\prime}}^{\prime}\right|=\operatorname{Bs}\left|N^{\prime}\right| \cap C_{0}^{\prime}$, contradicting our choice of $C_{0}^{\prime}$.

Now suppose that $b \geq a+1$. In this case we claim that we cannot have $k_{0}=k-2$, while $k_{0}=k-1$ occurs as gonality of some smooth curve in $|L|$ if and only if $b=a+1$. In fact, as in the proof of Corollary 2, if $k_{0}=k-2$ occurs then there exists a divisor $D_{0}$ such that $D_{0}^{2}=2$ and $D_{0} . L=k=2 a$. Now $D_{0} \sim F_{1}+F_{2}$ with $F_{i}>0, F_{i}^{2}=0, i=1,2, F_{1} \cdot F_{2}=1$, giving $2 \phi(L) \leq F_{1} . L+F_{2} . L=D_{0} . L=2 \phi(L)$, whence the contradiction $F_{1} \equiv E_{2} \equiv F_{2}$. Finally if $k_{0}=k-1$ occurs, then, as in the proof of Corollary 2 , there exists a divisor $D_{0}$ such that $D_{0}^{2}=2$ and $2 a=k \leq D_{0} . L \leq k+1=2 a+1$. Again $D_{0} \sim F_{1}+F_{2}$ with $F_{i}>0, F_{i}^{2}=0, i=1,2, F_{1} \cdot F_{2}=1$, giving $2 a \leq F_{1} \cdot L+F_{2} \cdot L=D_{0} \cdot L \leq 2 a+1$, which gives necessarily $F_{1} \equiv E_{1}, F_{2} \equiv E_{2}$, whence $b=a+1$.

Then, when $b \geq a+2$, minimal gonality and general gonality of $|L|$ coincide in this example, while, when $b=a+1$, the case $k_{0}=k-1$ occurs since, as above, we can choose a smooth curve $C_{0} \in|L|$ such that $x, y \in C_{0}$ and then $\left|\left(E_{1}+E_{2}\right)_{\mid C_{0}}(-x-y)\right|$ is a $g_{k-1}^{1}$.

Example 2. Let $\left|2 E_{i}\right|, i=1,2$, be two genus one pencils on an Enriques surface $S$ such that $E_{1} \cdot E_{2}=2$ and consider the line bundle

$$
L \sim a E_{1}+a E_{2} \text { for } a \geq 5 .
$$

By Lemma 2.14 we have that $\mu(L)=4 a-2<\min \left\{2 \phi(L),\left\lfloor\frac{L^{2}}{4}\right\rfloor+2\right\}$, whence $k:=$ gengon $|L|=4 a-2$ by Theorem 11. Also $\phi(L)=2 a$.

We claim that both $k_{0}=k-2$ and $k_{0}=k-1$ occur as gonality of some smooth curve in $|L|$. On any smooth curve $C \in|L|$ we have that $\left|\left(E_{1}+E_{2}\right)_{\mid C}\right|$ is a $g_{k+2}^{2}$ that cannot be very ample, whence there are two points $x, y \in C$ such that $\left|\left(E_{1}+E_{2}\right)_{\mid C}(-x-y)\right|$ is a $g_{k}^{1}$ on $C$. If $C$ is general in $|L|$ this series computes the gonality of $C$ by Theorem 11. On the other hand let $B_{1}, B_{2} \in\left|E_{1}+E_{2}\right|$ be two general smooth divisors and let $B_{1} \cap B_{2}=\left\{x_{1}, \ldots, x_{4}\right\}$. As in 
Example 1, we find that the general curves $C_{0} \in\left|\mathcal{J}_{\left\{x_{1}, \ldots, x_{4}\right\} / S} \otimes L\right|$ and $C_{0}^{\prime} \in\left|\mathcal{J}_{\left\{x_{1}, \ldots, x_{3}\right\} / S} \otimes L\right|$ are smooth and irreducible and that $\left|\left(E_{1}+E_{2}\right)_{\mid C_{0}}\left(-x_{1}-\ldots-x_{4}\right)\right|$ is a $g_{k-2}^{1}$ on $C_{0}$, while $\left|\left(E_{1}+E_{2}\right)_{\mid C_{0}^{\prime}}\left(-x_{1}-\ldots-x_{3}\right)\right|$ is a $g_{k-1}^{1}$ on $C_{0}^{\prime}$. Therefore the minimal gonality of a smooth curve in $|L|$ is $k_{0}:=\operatorname{gon}\left(C_{0}\right)=k-2$ by Corollary 2. The fact that $k_{0}^{\prime}:=\operatorname{gon}\left(C_{0}^{\prime}\right)=k-1$ can also be checked, with some cumbersome calculations.

\section{REFERENCES}

[ACGH] E. Arbarello, M. Cornalba, P. A. Griffiths, J. Harris. Geometry of Algebraic Curves, Volume I. Grundlehren der Mathematischen Wissenschaften 267. Springer-Verlag, New York, 1985.

[BEL] A. Bertram, L. Ein, R. Lazarsfeld. Surjectivity of Gaussian maps for line bundles of large degree on curves. Algebraic geometry (Chicago, IL, 1989), 15-25, LNM 1479. Springer, Berlin, 1991.

[BPV] W. Barth, C. Peters, A. van de Ven. Compact complex surfaces. Ergebnisse der Mathematik und ihrer Grenzgebiete 4. Springer-Verlag, Berlin-New York, 1984.

[Ca] G. Castelnuovo. Sulle superfici algebriche le cui sezioni iperpiane sono curve iperellittiche. Rend. Circ. Mat. Palermo 4, (1890) 73-88.

[CD] F. R. Cossec, I. V. Dolgachev. Enriques Surfaces I. Progress in Mathematics 76. Birkhäuser Boston, MA, 1989.

[Co1] F. R. Cossec. Projective models of Enriques surfaces. Math. Ann. 265, (1983) 283-334.

[Co2] F. R. Cossec. On the Picard group of Enriques surfaces. Math. Ann. 271, (1985) 577-600.

[CP] C. Ciliberto, G. Pareschi. Pencils of minimal degree on curves on a K3 surface. J. Reine Angew. Math. 460, (1995) 15-36.

[DM] R. Donagi, D. R. Morrison. Linear systems on K3-sections. J. Diff. Geom. 29, (1989) 49-64.

[En] F. Enriques. Sui sistemi lineari di ipersuperficie algebriche ad intersezioni variabili iperellittiche. Math. Ann. 46, (1895) 179-199.

[F] R. Friedman. Algebraic surfaces and holomorphic vector bundles. Universitext. Springer-Verlag, New York, 1998.

[Fa] M. L. Fania. Trigonal hyperplane sections of projective surfaces. Manuscr. Math. 68, (1990) 17-34.

[GL] M. Green, R. Lazarsfeld. Special divisors on curves on a K3 surface. Invent. Math. 89, (1987) 357-370.

[GLM] L. Giraldo, A. F. Lopez, R. Muñoz. On the projective normality of Enriques surfaces. Math. Ann. 324, (2002) 135-158.

[Ha] G. M. Hana. Projective models of Enriques surfaces in scrolls. Math. Nachr. 279, (2006) 242-254.

[Ki] H. Kim. Exceptional bundles on nodal Enriques surfaces. Manuscripta Math. 82, (1994) 1-13.

[KL1] A. L. Knutsen, A. F. Lopez. A sharp vanishing theorem for line bundles on K3 or Enriques surfaces. Proc. Amer. Math. Soc. 135, (2007) 3495-3498.

[KL2] A. L. Knutsen, A. F. Lopez. Brill-Noether theory of curves on Enriques surfaces II. In preparation.

[KL3] A. L. Knutsen, A. F. Lopez. Surjectivity of Gaussian maps for curves on Enriques surfaces. Adv. Geom. 7, (2007) 215-247.

[KLM] A. L. Knutsen, A. F. Lopez, R. Muñoz. On the extendability of projective surfaces and a genus bound for Enriques-Fano threefolds. Preprint math.AG/0605750.

[Kn] A. L. Knutsen. On kth order embeddings of K3 surfaces and Enriques surfaces. Manuscr. Math. 104, (2001) 211-237.

[La] R. Lazarsfeld. Brill-Noether-Petri without degenerations. J. Diff. Geom. 23, (1986) 299-307.

[Par] G. Pareschi. Exceptional linear systems on curves on Del Pezzo surfaces. Math. Ann. 291, (1991) $17-38$.

[Pao] R. Paoletti. Free pencils on divisors. Math. Ann. 303, (1995) 109-123.

[R] M. Reid. Special linear systems on curves lying on a K3 surface. J. London Math. Soc. 13, (1976) 454-458.

[Se] F. Serrano. Extension of morphisms defined on a divisor. Math. Ann. 277, (1987) 395-413.

[St] E. Stagnaro. Constructing Enriques surfaces from quintics in $\mathbb{P}_{K}^{3}$. Algebraic Geometry - open problems (Ravello, 1982). LNM 997. Springer, Berlin, 1983.

[SV] A. J. Sommese, A. Van de Ven. On the adjunction mapping. Math. Ann. 278, (1987) 593-603.

[T] A. N. Tyurin. Cycles, curves and vector bundles on an algebraic surface. Duke Math. J. 54, (1987) 1-26. 
[Um] Y. Umezu. Normal quintic surfaces which are birationally Enriques surfaces. Publ. Res. Inst. Math. Sci. 33, (1997) 359-384.

[Wa] J. Wahl. Introduction to Gaussian maps on an algebraic curve. Complex Projective Geometry, Trieste-Bergen 1989, London Math. Soc. Lecture Notes Ser. 179. CUP, Cambridge 1992, 304-323.

[Za] F. L. Zak. Some properties of dual varieties and their applications in projective geometry. Algebraic geometry (Chicago, IL, 1989), 273-280. LNM 479. Springer, Berlin, 1991.

Andreas Leopold Knutsen, Department of Mathematics, University of Bergen, Johannes Brunsgate 12, 5008 Bergen, Norway. E-Mail andreas.knutsen@math.uib.no

Angelo Felice Lopez, Dipartimento di Matematica, Università di Roma Tre, Largo San Leonardo Murialdo 1, 00146, Roma, ItALy. E-MAIL lopez@mat.uniroma3.it 